\title{
On the existence of solutions of a three steps crisis integro-differential equation
}

\author{
Dumitru Baleanu ${ }^{1,2^{*}}$, Khadijeh Ghafarnezhad ${ }^{3}$, Shahram Rezapour ${ }^{3,4}$ and Mehdi Shabibi ${ }^{5}$
}

${ }^{*}$ Correspondence:

dumitru@cankaya.edu.tr

${ }^{1}$ Department of Mathematics, Cankaya University, Ankara, Turkey

${ }^{2}$ Institute of Space Sciences, Bucharest, Romania

Full list of author information is available at the end of the article

\begin{abstract}
There are many natural phenomena including a crisis (such as a spate or contest) which could be described in three steps. We investigate the existence of solutions for a three step crisis integro-differential equation. We suppose that the second step is a point-wise defined singular fractional differential equation.
\end{abstract}

Keywords: Caputo derivative; Point-wise defined singular equation; Three steps crisis phenomena

\section{Introduction}

In most phenomena there appears usually a crisis. Our imagination as regards crises has effects on economy while there are distinct types of crisis-phenomena study in different fields of science such chemistry, social sciences, physics, mathematics, engineering and economy (see, for example, [1-7] and [8]). Considering the importance of modeling of crisis phenomena, some researchers are working and publishing in this area (see, for example, [9-13]). In 2016, Almeida, Bastos and Monteiro published a paper about modeling of some real phenomena by fractional differential equations [14]. As is well known, one of the best methods for mathematical describing this type phenomena is modeling of the problems as singular fractional integro-differential equations, which have been studied by researchers especially in recent decades (see, for example, [15-20] and [21]).

In 2010, Agarwal, O'Regan and Stanek investigated the existence of solutions for the problem $D^{\alpha} u(t)+f(t, u(t))=0$ with boundary conditions $u^{\prime}(0)=\cdots=u^{(n-1)}=0$ and $u(1)=\int_{0}^{1} u(s) d \mu(s)$, where $n \geq 2, \alpha \in(n-1, n), \mu(s)$ is a functional of bounded variation with $\int_{0}^{1} d \mu(s)<1$, and $f$ may have a singularity at $t=0$ [15]. They reviewed the existence of positive solutions for the system $D^{\alpha} u_{i}(t)+f_{i}\left(t, u_{1}(t), u_{2}(t)\right)=0$ with boundary conditions $u_{i}(0)=u_{i}^{\prime}(0)=0$ and $u_{i}(1)=\int_{0}^{1} u_{i}(t) d \eta(t)$ for $i=1,2$, where $t \in(0,1), \alpha \in(2,3]$, $\int_{0}^{1} u_{i}(t) d \eta(t)$ denotes the Riemann-Stieltjes integral, $f_{i} \in C\left([0,1] \times \mathbb{R}^{+} \times \mathbb{R}^{+}, \mathbb{R}\right)$ and $D^{\alpha}$ is the Riemann-Liouville fractional derivative of order $\alpha$ [16]. In 2013, Bai and Qui studied the singular problem $D^{\alpha} u+f\left(t, u, D^{\gamma} u, D^{\mu} u\right)+g\left(t, u, D^{\gamma} u, D^{\mu} u\right)=0$ with boundary conditions $u(0)=u^{\prime}(0)=u^{\prime \prime}(0)=u^{\prime \prime \prime}(0)=0$, where $3<\alpha<4,0<\gamma<1,1<\mu<2, D^{\alpha}$ is the Caputo fractional derivative and $f$ is a Caratheodory function on $[0,1] \times(0, \infty)^{3}$ [17]. Recently, the multi-singular point-wise defined fractional integro-differential equation $D^{\mu} x(t)+$ $f\left(t, x(t), x^{\prime}(t), D^{\beta} x(t), I^{p} x(t)\right)=0$ with boundary conditions $x^{\prime}(0)=x(\xi)$ and $x(1)=\int_{0}^{\eta} x(s) d s$ when $\mu \in[2,3)$ and $x^{\prime}(0)=x(\xi), x(1)=\int_{0}^{\eta} x(s) d s$ and $x^{(j)}(0)=0$ for $j=2, \ldots,[\mu]-1$ when

(c) The Author(s) 2018. This article is distributed under the terms of the Creative Commons Attribution 4.0 International License (http://creativecommons.org/licenses/by/4.0/), which permits unrestricted use, distribution, and reproduction in any medium, provided you give appropriate credit to the original author(s) and the source, provide a link to the Creative Commons license, and indicate if changes were made. 
$\mu \in[3, \infty)$ has been studied, where $0 \leq t \leq 1, x \in C^{1}[0,1], \mu \in[2, \infty), \beta, \xi, \eta \in(0,1), p>1$, $D^{\mu}$ is the Caputo fractional derivative of order $\mu$ and $f:[0,1] \times \mathbb{R}^{5} \rightarrow \mathbb{R}$ is a function such that $f(t, \cdot, \cdot, \cdot, \cdot)$ is singular at some points $t \in[0,1][19]$. By using these ideas and providing a new method for modeling of crisis phenomena, we investigate the existence of solutions for the point-wise defined three steps crisis integro-differential equation

$$
D^{\alpha} x(t)+f\left(t, x(t), x^{\prime}(t), D^{\beta} x(t), \int_{0}^{t} h(\xi) x(\xi) d \xi, \phi(x(t))\right)=0
$$

with boundary conditions $x(1)=x(0)=x^{\prime \prime}(0)=x^{n}(0)=0$, where $\alpha \geq 2, \lambda, \mu, \beta \in(0,1), \phi$ : $X \rightarrow X$ is a mapping such that $\|\phi(x)-\phi(y)\| \leq \theta_{0}\|x-y\|+\theta_{1}\left\|x^{\prime}-y^{\prime}\right\|$ for some nonnegative real numbers $\theta_{0}$ and $\theta_{1} \in[0, \infty)$ and all $x, y \in X, D^{\alpha}$ is the Caputo fractional derivative of order $\alpha, f\left(t, x_{1}(t), \ldots, x_{5}(t)\right)=f_{1}\left(t, x_{1}(t), \ldots, x_{5}(t)\right)$ for all $t \in[0, \lambda), f\left(t, x_{1}(t), \ldots, x_{5}(t)\right)=$ $f_{2}\left(t, x_{1}(t), \ldots, x_{5}(t)\right)$ for all $t \in[\lambda, \mu]$ and $f\left(t, x_{1}(t), \ldots, x_{5}(t)\right)=f\left(t, x_{1}(t), \ldots, x_{5}(t)\right)$ for all $t \in$ $(\mu, 1], f_{1}(t, \cdot, \cdot, \cdot, \cdot)$ and $f_{3}(t, \cdot, \cdot, \cdot, \cdot)$ are continuous on $[0, \lambda)$ and $(\mu, 1]$ and $f_{2}(t, \cdot, \cdot, \cdot, \cdot)$ is multisingular [19].

\section{Preliminaries}

Recall that $D^{\alpha} x(t)+f(t)=0$ is a point-wise defined equation on $[0,1]$ if there exists a set $E \subset[0,1]$ such that the measure of $E^{c}$ is zero and the equation holds on $E$ [19]. In this paper, we use $\|\cdot\|_{1}$ for the norm of $L^{1}[0,1],\|\cdot\|$ for the sup norm of $Y=C[0,1]$ and $\|x\|_{*}=$ $\max \left\{\|x\|,\left\|x^{\prime}\right\|\right\}$ for the norm of $X=C^{1}[0,1]$. As is well known, the Riemann-Liouville integral of order $p$ with the lower limit $a \geq 0$ for a function $f:(a, \infty) \rightarrow \mathbb{R}$ is defined by $I_{a^{+}}^{p} f(t)=\frac{1}{\Gamma(p)} \int_{a}^{t}(t-s)^{p-1} f(s) d s$, provided that the right-hand side is point-wise defined on $(a, \infty)$ [22]. We denote $I_{0^{+}}^{p} f(t)$ by $I^{p} f(t)$. Also, the Caputo fractional derivative of order $\alpha>0$ is defined by ${ }^{c} D^{\alpha} f(t)=\frac{1}{\Gamma(n-\alpha)} \int_{0}^{t} \frac{f^{n}(s)}{(t-s)^{\alpha+1-n}} d s$, where $n=[\alpha]+1$ and $f:(a, \infty) \rightarrow \mathbb{R}$ is a function [22]. Let $\Psi$ be the family of nondecreasing functions $\psi:[0, \infty) \rightarrow[0, \infty)$ such that $\sum_{n=1}^{\infty} \psi^{n}(t)<\infty$ for all $t>0$ (see [23]). One can check that $\psi(t)<t$ for all $t>0$. Let $(X, d)$ be a metric space and $T: X \rightarrow X$ and $\alpha: X \times X \rightarrow[0, \infty)$ two maps. Then $T$ is called an $\alpha$-admissible map whenever $\alpha(x, y) \geq 1$ implies $\alpha(T x, T y) \geq 1$ [23]. The map $T$ is called an $\alpha$-admissible map whenever $\alpha(x, y) \geq 1$ implies $\alpha(T x, T y) \geq 1$ [23]. Let $(X, d)$ be a metric space, $\psi \in \Psi$ and $\alpha: X \times X \rightarrow[0, \infty)$ a map. A self-map $T: X \rightarrow X$ is called an $\alpha$ - $\psi$-contraction whenever $\alpha(x, y) d(T x, T y) \leq \psi(d(x, y))$ for all $x, y \in X$ [23]. To prove the existence of solutions, we need next results.

Lemma 2.1 ([23]) Let $(X, d)$ be a complete metric space, $\psi \in \Psi, \alpha: X \times X \rightarrow[0, \infty)$ a map and $T: X \rightarrow X$ an $\alpha$-admissible $\alpha$ - $\psi$-contraction. If $T$ is continuous and there exists $x_{0} \in X$ such that $\alpha\left(x_{0}, T x_{0}\right) \geq 1$, then $T$ has a fixed point.

Lemma 2.2 ([24] Let $n-1 \leq \alpha<n$ and $x \in C(0,1) \cap L^{1}(0,1)$. Then we have $I^{\alpha} D^{\alpha} x(t)=$ $x(t)+\sum_{i=0}^{n-1} c_{i} t^{i}$ for some real constants $c_{0}, \ldots, c_{n-1}$.

Lemma 2.3 ([21]) Let $\beta>0$ and $\alpha>-1$. Then $\int_{0}^{t}(t-s)^{\alpha-1} s^{\beta} d s=B(\beta+1, \alpha) t^{\alpha+\beta}$, where $B(\beta, \alpha)=\frac{\Gamma(\alpha) \Gamma(\beta)}{\Gamma(\alpha+\beta)}$.

Lemma 2.4 ([25]) Let $E$ be a Banach space, $P \subseteq E$ a cone and $\Omega_{1}, \Omega_{2}$ two bounded open balls of E centered at the origin with $\overline{\Omega_{1}} \subset \Omega_{2}$. Suppose that $F: P \cap\left(\bar{\Omega}_{2} \backslash \Omega_{1}\right) \rightarrow P$ is a completely continuous operator such that either 
(i. $\left.i_{1}\right)\|F(x)\| \leq\|x\|$ for all $x \in P \cap \partial \Omega_{1}$ and $\|F x\| \geq\|x\|$ for all $x \in P \cap \partial \Omega_{2}$, or

$\left(i_{2}\right)\|F x\| \geq\|x\|$ for all $x \in P \cap \partial \Omega_{1}$ and $\|F x\| \leq\|x\|$ for all $x \in P \cap \partial \Omega_{2}$

holds. Then $F$ has a fixed point in $P \cap\left(\Omega_{2} \backslash \Omega_{1}\right)$.

\section{Main results}

Now, we are ready for providing our results.

Lemma 3.1 Let $\alpha \geq 2, n=[\alpha]+1$ and $f \in L^{1}[0,1]$. A map $u$ is a solution for the pointwise defined equation $D^{\alpha} x(t)+f(t)=0$ with boundary conditions $x^{\prime}(1)=x(0)=x^{\prime \prime}(0)=$ $\cdots=x^{n-1}(0)=0$ if and only if $u(t)=\int_{0}^{1} G(t, s) f(s) d s$ for all $t \in[0,1]$, where $G(t, s)=\frac{t(1-s)^{\alpha-2}}{\Gamma(\alpha-1)}$ whenever $0 \leq t \leq s \leq 1$ and $G(t, s)=\frac{t(1-s)^{\alpha-2}}{\Gamma(\alpha-1)}-\frac{(t-s)^{\alpha-1}}{\Gamma(\alpha)}$ whenever $0 \leq s \leq t \leq 1$.

Proof Let $E$ be a subset of $[0,1]$ such that $m\left(E^{c}\right)=0$ and $D^{\alpha} x(t)+f(t)=0$ for all $t \in E$. Here, $m$ is the Lebesgue measure on $\mathbb{R}$. Note that $E$ is dense in $[0,1]$. Let $f_{0} \in C[0,1]$ be a function such that $f_{0}=f$ on $E$. Then we have

$$
\begin{aligned}
I^{\alpha}(f(t)) & =\frac{1}{\Gamma(\alpha)} \int_{0}^{t}(t-s)^{\alpha-1} f(s) d s \\
& =\frac{1}{\Gamma(\alpha)}\left(\int_{[0, t] \cap E}(t-s)^{\alpha-1} f(s) d s+\int_{[0, t] \cap E^{c}}(t-s)^{\alpha} f(s) d s\right) \\
& =\frac{1}{\Gamma(\alpha)} \int_{[0, t] \cap E}(t-s)^{\alpha-1} f_{0}(s) d s \\
& =\frac{1}{\Gamma(\alpha)}\left(\int_{[0, t] \cap E}(t-s)^{\alpha-1} f_{0}(s) d s+\int_{[0, t] \cap E^{c}}(t-s)^{\alpha-1} f_{0}(s) d s\right) \\
& =\frac{1}{\Gamma(\alpha)} \int_{0}^{t}(t-s)^{\alpha-1} f_{0}(s) d s=I^{\alpha}\left(f_{0}(t)\right)
\end{aligned}
$$

for all $t \in E$. Let $t \in E^{c} \backslash\{0\}$. Choose a sequence $\left\{t_{n}\right\}_{n \geq 1}$ in $E$ such that $t_{n} \rightarrow t^{-}$. Then

$$
\begin{aligned}
I^{\alpha}(f(t)) & =\frac{1}{\Gamma(\alpha)} \int_{0}^{t}(t-s)^{\alpha-1} f(s) d s \\
& =\lim _{n \rightarrow \infty} \frac{1}{\Gamma(\alpha)} \int_{0}^{t_{n}}\left(t_{n}-s\right)^{\mu-1} f(s) d s=\lim _{n \rightarrow \infty} I^{\alpha}\left(f\left(t_{n}\right)\right) \\
& =\lim _{n \rightarrow \infty} I^{\alpha}\left(f_{0}\left(t_{n}\right)\right)=\lim _{n \rightarrow \infty} \frac{1}{\Gamma(\alpha)} \int_{0}^{t_{n}}\left(t_{n}-s\right)^{\alpha-1} f_{0}(s) d s \\
& =\frac{1}{\Gamma(\alpha)} \int_{0}^{t}(t-s)^{\alpha-1} f(s) d s \\
& =I^{\alpha}\left(f_{0}(t)\right) .
\end{aligned}
$$

For $t=0 \in E^{c}$, we get $I^{\alpha}(f(t))=I^{\alpha}\left(f_{0}(t)\right)=0$ and so $I^{\alpha}(f(t))=I^{\alpha}\left(f_{0}(t)\right)$ for all $t \in[0,1]$. Thus, the equation $D^{\alpha} x(t)+f(t)=0$ equivalents to $I^{\alpha}\left(D^{\alpha} x(t)\right)=I^{\alpha}\left(-f_{0}(t)\right)$ on $[0,1]$. By using Lemma 2.2 and the boundary condition, we get $x(t)=-\frac{1}{\Gamma(\alpha)} \int_{0}^{t}(t-s)^{\alpha-1} y(s) d s+c_{1} t$ and so $x^{\prime}(t)=-\frac{1}{\Gamma(\alpha-1)} \int_{0}^{t}(t-s)^{\alpha-2} y(s) d s+c_{1}$. Hence, $x^{\prime}(1)=-\frac{1}{\Gamma(\alpha-1)} \int_{0}^{1}(1-s)^{\alpha-2} y(s) d s+c_{1}$. Since $x^{\prime}(1)=0, c_{1}=\frac{1}{\Gamma(\alpha-1)} \int_{0}^{1}(1-s)^{\alpha-2} y(s) d s$ and so

$$
x(t)=-\frac{1}{\Gamma(\alpha)} \int_{0}^{t}(t-s)^{\alpha-1} y(s) d s+\frac{t}{\Gamma(\alpha-1)} \int_{0}^{1}(1-s)^{\alpha-2} y(s) d s=\int_{0}^{1} G(t, s) y(s) d s,
$$


where $G(t, s)=\frac{t(1-s)^{\alpha-2}}{\Gamma(\alpha-1)}$ whenever $0 \leq t \leq s \leq 1$ and $G(t, s)=\frac{t(1-s)^{\alpha-2}}{\Gamma(\alpha-1)}-\frac{(t-s)^{\alpha-1}}{\Gamma(\alpha)}$ whenever $0 \leq s \leq t \leq 1$. Also, an easy calculation shows that $u(t)=\int_{0}^{1} G(t, s) f(s) d s$ is a solution for the equation with the boundary conditions. This completes the proof.

Note that for the Green function $G(t, s)$ in the last result we have $G(t, s) \geq \frac{(\alpha-2)|t-s|^{\alpha-1}}{\Gamma(\alpha)} \geq 0$, $G(t, s) \leq \frac{t(1-)^{\alpha-2}}{\Gamma(\alpha-1)}, \frac{\partial}{\partial t} G(t, s) \geq 0$ and $\frac{\partial}{\partial t} G(t, s) \leq \frac{(1-s)^{\alpha-2}}{\Gamma(\alpha-1)}$ for all $t, s \in[0,1]$. Also, $G$ and $\frac{\partial}{\partial t} G$ are continuous with respect to $t$. Consider the space $X=C^{1}[0,1]$ with the norm $\|\cdot\|_{*}$, where $\|x\|_{*}=\max \left\{\|x\|,\left\|x^{\prime}\right\|\right\}$ and $\|\cdot\|$ is the supremum norm on $C[0,1]$. Let $\lambda, \mu \in(0,1)$ with $\lambda<\mu$. Suppose that $f_{1}$ and $f_{3}$ are continuous functions (with respect to the first variable) on $[0, \lambda] \times X^{5}$ and $[\mu, 1] \times X^{5}$, respectively, and $f_{2}$ is a function on $(\lambda, \mu) \times X^{5}$ which is singular at some points $t \in(\lambda, \mu)$. Let $f$ be a map on $[0,1] \times X^{5}$ such that $\left.f\right|_{[0, \lambda] \times X^{5}}=f_{1}$, $\left.f\right|_{(\lambda, \mu) \times X^{5}}=f_{2}$ and $\left.f\right|_{[\mu, 0] \times X^{5}}=f_{3}$. We denote this case briefly by $\left[\lambda, \mu, f=\left(f_{1}, f_{2}, f_{3}\right)\right]$. Define the map $F: X \rightarrow X$ by $F_{x}(t)=\int_{0}^{1} G(t, s) f\left(s, x(s), x^{\prime}(s), D^{\beta} x(s), \int_{0}^{s} h(\xi) x(\xi) d \xi, \phi(x(s))\right) d s$ for all $t \in[0,1]$. Note that the singular point-wise defined equation (1) has a solution $u_{0} \in X$ if and only if $u_{0}$ a fixed point of the map $F$.

Theorem 3.2 Let $\left[\lambda, \mu, f=\left(f_{1}, f_{2}, f_{3}\right)\right]$ with $f_{1}(s, 0,0,0,0,0)=f_{3}(t, 0,0,0,0,0)=0$ for all $s \in[0, \lambda]$ and $t \in[\mu, 1]$. Assume that there exist two maps $H: X^{5} \rightarrow[0, \infty)$ and $\Phi:$ $(\lambda, \mu) \rightarrow[0, \infty)$ such that $f_{2}\left(t, x_{1}, x_{2}, \ldots, x_{5}\right) \leq \Phi(t) H\left(x_{1}, x_{2}, \ldots, x_{5}\right)$ for all $\left(x_{1}, \ldots, x_{5}\right) \in X^{5}$ and almost all $t \in(\lambda, \mu)$, where $H: X^{5} \rightarrow[0, \infty)$ is nondecreasing with respect to all its components, $\int_{\lambda}^{\mu}(1-s)^{\alpha-1} \Phi(s) d s<\infty$ and $\lim _{z \rightarrow 0^{+}} \frac{H(z, z, z, z, z)}{z}=0$. Suppose that the map $q$ defined by $q(t)=\lim _{\max \left\{\left\|x_{1}\right\|, \ldots,\left\|x_{5}\right\|\right\} \rightarrow \infty} \frac{f_{2}\left(t, x_{1} x_{2}, \ldots, x_{5}\right)}{\max \left\{\left\|x_{1}\right\|, \ldots,\left\|x_{5}\right\|\right\}}$ for almost all $t \in(\lambda, \mu)$ has the property that $\frac{\alpha-2}{\Gamma(\alpha)} \int_{\lambda}^{\mu}(\mu-s)^{\alpha-2} q(s) d s>1$. Assume that there exist nonnegative real numbers $l_{1}, \ldots, l_{5}, l_{1}^{\prime}, \ldots, l_{5}^{\prime}$ and mappings $a_{1}, \ldots, a_{5}:(\lambda, \mu) \rightarrow[0, \infty)$ and $\Lambda_{1}, \ldots, \Lambda_{5}: X^{5} \rightarrow[0, \infty)$ such that $\left|f_{1}\left(t, x_{1}, \ldots, x_{5}\right)-f_{1}\left(t, y_{1}, \ldots, y_{5}\right)\right| \leq \sum_{i=1}^{5} l_{i}\left|x_{i}-y_{i}\right|$,

$$
\left|f_{2}\left(t, x_{1}, \ldots, x_{5}\right)-f_{2}\left(t, y_{1}, \ldots, y_{5}\right)\right| \leq \sum_{i=1}^{5} a_{i}(t) \Lambda_{i}\left(\left|x_{1}-y_{1}\right|, \ldots,\left|x_{5}-y_{5}\right|\right)
$$

and $\left|f_{3}\left(t, x_{1}, \ldots, x_{5}\right)-f_{3}\left(t, y_{1}, \ldots, y_{5}\right)\right| \leq \sum_{i=1}^{5} l_{i}^{\prime}\left|x_{i}-y_{i}\right|$ for all $t$ and $x_{1}, \ldots, x_{5} \in X$. If $\lim _{z \rightarrow 0^{+}} \frac{\Lambda_{i}(z, z, z, z, z)}{z}=q_{i}<\infty$ and $\left[\frac{L\left(1-(1-\lambda)^{\alpha-1}\right)}{\Gamma(\alpha)}+\frac{L^{\prime}}{\Gamma(\alpha)}(1-\mu)^{\alpha-1}\right]<1$ for $i=1, \ldots, 5$, where $m_{0}=$ $\int_{0}^{1}|h(\xi)| d \xi, L=l_{1}+l_{2}+\frac{l_{3}}{\Gamma(2-\beta)}+m_{0} l_{4}+\theta_{0} l_{5}+\theta_{1} l_{5}$ and $L^{\prime}=l_{1}^{\prime}+l_{2}^{\prime}+\frac{l_{3}^{\prime}}{\Gamma(2-\beta)}+m_{0} l_{4}^{\prime}+\theta_{0} l_{5}^{\prime}+\theta_{1} l_{5}^{\prime}$, then the problem (1) has a solution.

Proof Consider the closed cone $P=\left\{x \in X: x(t) \geq 0\right.$ and $x^{\prime}(t) \geq 0$ for all $\left.t \in[0,1]\right\}$ in $X$. Let $\epsilon>0$ be given, $\left\{x_{n}\right\}_{n \geq 1}$ a sequence in $X$ with $x_{n} \rightarrow x$. Choose a natural number $N$ such that $\left\|x_{n}-x\right\|<\epsilon$ for all $n \geq N$. Take $\epsilon>0$ such that

$$
\left[\frac{L\left(1-(1-\lambda)^{\alpha-1}\right)}{\Gamma(\alpha)}+\frac{\left(q_{i}+\epsilon\right) \epsilon}{\Gamma(\alpha-1)} \sum_{i=1}^{5} M_{i}(\lambda, \mu)+\frac{L^{\prime}}{\Gamma(\alpha)}(1-\mu)^{\alpha-1}\right]<1
$$

for $i=1, \ldots, 5$, where $M_{i}(\lambda, \mu)=\int_{\lambda}^{\mu}(1-s)^{\alpha-2} a_{i}(s) d s$. Note that

$$
\begin{aligned}
\left|F_{x_{n}}(t)-F_{x}(t)\right| \leq & \int_{0}^{\lambda} G(t, s) \mid f_{1}\left(s, x_{n}(s), x_{n}^{\prime}(s), D^{\beta} x_{n}(s), \int_{0}^{s} h(\xi) x_{n}(\xi) d \xi, \phi\left(x_{n}(s)\right)\right) \\
& -f_{1}\left(s, x(s), x^{\prime}(s), D^{\beta} x(s), \int_{0}^{s} h(\xi) x(\xi) d \xi, \phi(x(s))\right) \mid d s
\end{aligned}
$$




$$
\begin{aligned}
& +\int_{\lambda}^{\mu} G(t, s) \mid f_{2}\left(s, x_{n}(s), x_{n}^{\prime}(s), D^{\beta} x_{n}(s), \int_{0}^{s} h(\xi) x_{n}(\xi) d \xi, \phi\left(x_{n}(s)\right)\right) \\
& -f_{2}\left(s, x(s), x^{\prime}(s), D^{\beta} x(s), \int_{0}^{s} h(\xi) x(\xi) d \xi, \phi(x(s))\right) \mid d s \\
& +\int_{\mu}^{1} G(t, s) \mid f_{3}\left(s, x_{n}(s), x_{n}^{\prime}(s), D^{\beta} x_{n}(s), \int_{0}^{s} h(\xi) x_{n}(\xi) d \xi, \phi\left(x_{n}(s)\right)\right) \\
& -f_{3}\left(s, x(s), x^{\prime}(s), D^{\beta} x(s), \int_{0}^{s} h(\xi) x(\xi) d \xi, \phi(x(s))\right) \mid d s \\
& \leq \int_{0}^{\lambda} G(t, s)\left(l_{1}\left|x_{n}(s)-x(s)\right|+l_{2}\left|x_{n}^{\prime}(s)-x^{\prime}(s)\right|+l_{3}\left|D^{\beta}\left(x_{n}-x\right)(s)\right|\right. \\
& \left.+l_{4} \int_{0}^{s}\left|x_{n}(\xi)-x(\xi)\right| d \xi+l_{5}\left|\phi\left(x_{n}(s)-x(s)\right)\right|\right) \\
& +\int_{\lambda}^{\mu} G(t, s)\left(a _ { 1 } ( s ) \Lambda _ { 1 } \left(\left|x_{n}(s)-x(s)\right|,\left|x_{n}^{\prime}(s)-x^{\prime}(s)\right|,\right.\right. \\
& \left.\left|D^{\beta}\left(x_{n}-x\right)(s)\right|, \int_{0}^{s}\left|x_{n}(\xi)-x(\xi)\right| d \xi,\left|\phi\left(x_{n}(s)-x(s)\right)\right|\right) \\
& +\cdots+a_{5}(s) \Lambda_{5}\left(\left|x_{n}(s)-x(s)\right|,\left|x_{n}^{\prime}(s)-x^{\prime}(s)\right|,\right. \\
& \left.\left|D^{\beta}\left(x_{n}-x\right)(s)\right|, \int_{0}^{s}\left|x_{n}(\xi)-x(\xi)\right| d \xi,\left|\phi\left(x_{n}(s)-x(s)\right)\right|\right) \\
& +\int_{\mu}^{1} G(t, s)\left(l_{1}^{\prime}\left|x_{n}(s)-x(s)\right|+l_{2}^{\prime}\left|x_{n}^{\prime}(s)-x^{\prime}(s)\right|+l_{3}^{\prime}\left|D^{\beta}\left(x_{n}-x\right)(s)\right|\right. \\
& \left.+l_{4}^{\prime} \int_{0}^{s}\left|x_{n}(\xi)-x(\xi)\right| d \xi+l_{5}^{\prime}\left|\phi\left(x_{n}(s)-x(s)\right)\right|\right) \\
& \leq \int_{0}^{\lambda} G(t, s)\left(l_{1}\left\|x_{n}-x\right\|+l_{2}\left\|x_{n}^{\prime}-x^{\prime}\right\|+\frac{l_{3}}{\Gamma(2-\beta)}\left\|x_{n}^{\prime}-x^{\prime}\right\|\right. \\
& \left.+m_{0} l_{4}\left\|x_{n}-x\right\|+\theta_{0} l_{5}\left\|x_{n}-x\right\|+\theta_{1} l_{5}\left\|x_{n}^{\prime}-x^{\prime}\right\|\right) \\
& +\int_{\lambda}^{\mu} G(t, s)\left(a _ { 1 } ( s ) \Lambda _ { 1 } \left(\left\|x_{n}-x\right\|,\left\|x_{n}^{\prime}-x^{\prime}\right\|, \frac{1}{\Gamma(2-\beta)}\left\|x_{n}^{\prime}-x^{\prime}\right\|,\right.\right. \\
& \left.m_{0}\left\|x_{n}-x\right\|, \theta_{0} l_{5}\left\|x_{n}-x\right\|+\theta_{1} l_{5}\left\|x_{n}^{\prime}-x^{\prime}\right\|\right) \\
& +\cdots+a_{5}(s) \Lambda_{1}\left(\left\|x_{n}-x\right\|,\left\|x_{n}^{\prime}-x^{\prime}\right\|, \frac{1}{\Gamma(2-\beta)}\left\|x_{n}^{\prime}-x^{\prime}\right\|,\right. \\
& \left.m_{0}\left\|x_{n}-x\right\|, \theta_{0} l_{5}\left\|x_{n}-x\right\|+\theta_{1} l_{5}\left\|x_{n}^{\prime}-x^{\prime}\right\|\right) d s \\
& +\int_{\mu}^{1} G(t, s)\left(l_{1}^{\prime}\left\|x_{n}-x\right\|+l_{2}^{\prime}\left\|x_{n}^{\prime}-x^{\prime}\right\|+\frac{l_{3}^{\prime}}{\Gamma(2-\beta)}\left\|x_{n}^{\prime}-x^{\prime}\right\|\right. \\
& \left.+m_{0} l_{4}^{\prime}\left\|x_{n}-x\right\|+\theta_{0} l_{5}^{\prime}\left\|x_{n}-x\right\|+\theta_{1} l_{5}^{\prime}\left\|x_{n}^{\prime}-x^{\prime}\right\|\right) d s \\
& \leq\left(l_{1}+l_{2}+\frac{l_{3}}{\Gamma(2-\beta)}+l_{4} m_{0}+l_{5} \theta_{0}+l_{5} \theta_{1}\right)\left\|x_{n}-x\right\|_{*} \int_{0}^{\lambda} G(t, s) d s
\end{aligned}
$$




$$
\begin{aligned}
& +\int_{\lambda}^{\mu} G(t, s)\left(\sum _ { i = 1 } ^ { 5 } a _ { i } ( s ) \Lambda _ { i } \left(l\left\|x_{n}-x\right\|_{*}, l\left\|x_{n}-x\right\|_{*}, l\left\|x_{n}-x\right\|_{*},\right.\right. \\
& \left.l\left\|x_{n}-x\right\|_{*}, l\left\|x_{n}-x\right\|_{*}\right) d s \\
& +\left(l_{1}^{\prime}+l_{2}^{\prime}+\frac{l_{3}^{\prime}}{\Gamma(2-\beta)}+l_{4}^{\prime} m_{0}+l_{5}^{\prime} \theta_{0}+l_{5}^{\prime} \theta_{1}\right)\left\|x_{n}-x\right\|_{*} \int_{\mu}^{1} G(t, s) d s
\end{aligned}
$$

for all $t \in[0,1]$, where $l=\max \left\{1, \frac{1}{\Gamma(2-\beta)}, m_{0}, \theta_{0}+\theta_{1}\right\}$. For each $1 \leq i \leq 5$ choose $0<\delta_{i}(\epsilon)<$ $\epsilon^{2}$ such that $\frac{\Lambda_{i}(z, z, z, z, z)}{z}<q_{i}+\epsilon$ for all $z \in\left(0, \delta_{i}(\epsilon)\right]$. Thus, $\Lambda_{i}(z, z, z, z, z)<\left(q_{i}+\epsilon\right) z$ for all $z \in\left(0, \delta_{i}(\epsilon)\right]$ and $1 \leq i \leq 5$. Put $\delta:=\min _{1 \leq i \leq 5} \delta_{i}(\epsilon)$. Then we have

$$
\Lambda_{i}(\delta, \delta, \delta, \delta, \delta)<\left(q_{i}+\epsilon\right) \delta<\left(q_{i}+\epsilon\right) \epsilon^{2}
$$

Let $m_{1}$ be a natural number such that $l\left\|x_{n}-x\right\|_{*}<\delta$ for all $n \geq m_{1}$. This implies that $\Lambda_{i}\left(l\left\|x_{n}-x\right\|_{*}, \ldots, l\left\|x_{n}-x\right\|_{*}\right)<\Lambda_{i}(\delta, \delta, \delta, \delta, \delta)<\left(q_{i}+\epsilon\right) \epsilon^{2}$ for all $n \geq m_{1}$ and $i=1, \ldots, 5$.

Thus,

$$
\begin{aligned}
\left|F_{x_{n}}(t)-F_{x}(t)\right| \leq & L\left\|x_{n}-x\right\|_{*} \int_{0}^{\lambda} G(t, s) d s \\
& +\left(q_{i}+\epsilon\right) \epsilon^{2} \int_{\lambda}^{\mu} G(t, s) \sum_{i=1}^{5} a_{i}(s) d s+L^{\prime}\left\|x_{n}-x\right\|_{*} \int_{\mu}^{1} G(t, s) d s
\end{aligned}
$$

for all $n \geq \max \left\{N, m_{1}\right\}$. This implies that

$$
\begin{aligned}
& \left|F_{x_{n}}(t)-F_{x}(t)\right| \\
& \leq \frac{L \epsilon t}{\Gamma(\alpha-1)} \int_{0}^{\lambda}(1-s)^{\alpha-2} d s \\
& \quad+\frac{\left(q_{i}+\epsilon\right) \epsilon^{2} t}{\Gamma(\alpha-1)} \sum_{i=1}^{5} \int_{\lambda}^{\mu}(1-s)^{\alpha-2} a_{i}(s) d s+\frac{L^{\prime} \epsilon t}{\Gamma(\alpha-1)} \int_{\mu}^{1}(1-s)^{\alpha-2} d s \\
& =\frac{L \epsilon t\left(1-(1-\lambda)^{\alpha-1}\right)}{\Gamma(\alpha)}+\frac{\left(q_{i}+\epsilon\right) \epsilon^{2} t}{\Gamma(\alpha-1)} \sum_{i=1}^{5} M_{i}(\lambda, \mu)+\frac{L^{\prime} \epsilon t}{\Gamma(\alpha)}(1-\mu)^{\alpha-1}
\end{aligned}
$$

for all $n \geq \max \left\{N, m_{1}\right\}$ and $t \in[0,1]$ and so

$$
\left\|F_{x_{n}}-F_{x}\right\| \leq\left[\frac{L\left(1-(1-\lambda)^{\alpha-1}\right)}{\Gamma(\alpha)}+\frac{\left(q_{i}+\epsilon\right) \epsilon}{\Gamma(\alpha-1)} \sum_{i=1}^{5} M_{i}(\lambda, \mu)+\frac{L^{\prime}}{\Gamma(\alpha)}(1-\mu)^{\alpha-1}\right] \epsilon<\epsilon .
$$

By using similar calculations, we get

$$
\begin{aligned}
\left|F_{x_{n}}^{\prime}(t)-F_{x}^{\prime}(t)\right| \leq & \int_{0}^{\lambda} \frac{\partial G}{\partial t}(t, s) \mid f_{1}\left(s, x_{n}(s), x_{n}^{\prime}(s), D^{\beta} x_{n}(s), \int_{0}^{s} h(\xi) x_{n}(\xi) d \xi, \phi\left(x_{n}(s)\right)\right) \\
& -f_{1}\left(s, x(s), x^{\prime}(s), D^{\beta} x(s), \int_{0}^{s} h(\xi) x(\xi) d \xi, \phi(x(s))\right) \mid d s \\
& +\int_{\lambda}^{\mu} \frac{\partial G}{\partial t}(t, s) \mid f_{2}\left(s, x_{n}(s), x_{n}^{\prime}(s), D^{\beta} x_{n}(s), \int_{0}^{s} h(\xi) x_{n}(\xi) d \xi, \phi\left(x_{n}(s)\right)\right)
\end{aligned}
$$




$$
\begin{aligned}
& -f_{2}\left(s, x(s), x^{\prime}(s), D^{\beta} x(s), \int_{0}^{s} h(\xi) x(\xi) d \xi, \phi(x(s))\right) \mid d s \\
& +\int_{\mu}^{1} \frac{\partial G}{\partial t}(t, s) \mid f_{3}\left(s, x_{n}(s), x_{n}^{\prime}(s), D^{\beta} x_{n}(s), \int_{0}^{s} h(\xi) x_{n}(\xi) d \xi, \phi\left(x_{n}(s)\right)\right) \\
& -f_{3}\left(s, x(s), x^{\prime}(s), D^{\beta} x(s), \int_{0}^{s} h(\xi) x(\xi) d \xi, \phi(x(s))\right) \mid d s \\
& \leq \int_{0}^{\lambda} \frac{\partial G}{\partial t}(t, s)\left(l_{1}\left\|x_{n}-x\right\|+l_{2}\left\|x_{n}^{\prime}-x^{\prime}\right\|+\frac{l_{3}}{\Gamma(2-\beta)}\left\|x_{n}^{\prime}-x^{\prime}\right\|\right. \\
& \left.+m_{0} l_{4}\left\|x_{n}-x\right\|+\theta_{0} l_{5}\left\|x_{n}-x\right\|+\theta_{1} l_{5}\left\|x_{n}^{\prime}-x^{\prime}\right\|\right) d s \\
& +\int_{\lambda}^{\mu} \frac{\partial G}{\partial t}(t, s)\left(a _ { 1 } ( s ) \Lambda _ { 1 } \left(\left\|x_{n}-x\right\|,\left\|x_{n}^{\prime}-x^{\prime}\right\|, \frac{1}{\Gamma(2-\beta)}\left\|x_{n}^{\prime}-x^{\prime}\right\|,\right.\right. \\
& \left.m_{0}\left\|x_{n}-x\right\|, \theta_{0} l_{5}\left\|x_{n}-x\right\|+\theta_{1} l_{5}\left\|x_{n}^{\prime}-x^{\prime}\right\|\right) \\
& +\cdots+a_{5}(s) \Lambda_{5}\left(\left\|x_{n}-x\right\|,\left\|x_{n}^{\prime}-x^{\prime}\right\|, \frac{1}{\Gamma(2-\beta)}\left\|x_{n}^{\prime}-x^{\prime}\right\|,\right. \\
& \left.m_{0}\left\|x_{n}-x\right\|, \theta_{0} l_{5}\left\|x_{n}-x\right\|+\theta_{1} l_{5}\left\|x_{n}^{\prime}-x^{\prime}\right\|\right) d s \\
& +\int_{\mu}^{1} \frac{\partial G}{\partial t}(t, s)\left(l_{1}^{\prime}\left\|x_{n}-x\right\|+l_{2}^{\prime}\left\|x_{n}^{\prime}-x^{\prime}\right\|+\frac{l_{3}^{\prime}}{\Gamma(2-\beta)}\left\|x_{n}^{\prime}-x^{\prime}\right\|\right. \\
& \left.+m_{0} l_{4}^{\prime}\left\|x_{n}-x\right\|+\theta_{0} l_{5}^{\prime}\left\|x_{n}-x\right\|+\theta_{1} l_{5}^{\prime}\left\|x_{n}^{\prime}-x^{\prime}\right\|\right) d s \\
& \leq\left[\frac{L\left(1-(1-\lambda)^{\alpha-1}\right)}{\Gamma(\alpha)}+\frac{\left(q_{i}+\epsilon\right) \epsilon}{\Gamma(\alpha-1)} \sum_{i=1}^{5} M_{i}(\lambda, \mu)+\frac{L^{\prime}}{\Gamma(\alpha)}(1-\mu)^{\alpha-1}\right] \epsilon
\end{aligned}
$$

for all $n \geq \max \left\{N, m_{1}\right\}$ and $t \in[0,1]$. Hence, $\left\|F_{x_{n}}^{\prime}-F_{x}^{\prime}\right\| \leq \epsilon$ for sufficiently large $n$ and so $\left\|F_{x_{n}}-F_{x}\right\|_{*}=\max \left\{\left\|F_{x_{n}}-F_{x}\right\|,\left\|F_{x_{n}}^{\prime}-F_{x}^{\prime}\right\|\right\}<\epsilon$ for sufficiently large $n$. This implies that $F_{x_{n}} \rightarrow F_{x}$ in $X$. Now, we prove that $F$ maps bounded sets into bounded sets of $X$. Let $M$ be a bounded set of $X$. Choose $r>0$ such that $\|x\|_{*}<r$ for all $x \in M$. Let $x \in M$. Then

$$
\begin{aligned}
\left|F_{x}(t)\right| \leq & \mid \int_{0}^{\lambda} G(t, s) f_{1}\left(s, x(s), x^{\prime}(s), D^{\beta} x(s), \int_{0}^{s} h(\xi) x(\xi) d \xi, \phi(x(s))\right) d s \\
& +\int_{\lambda}^{\mu} G(t, s) f_{2}\left(s, x(s), x^{\prime}(s), D^{\beta} x(s), \int_{0}^{s} h(\xi) x(\xi) d \xi, \phi(x(s))\right) d s \\
& +\int_{\mu}^{1} G(t, s) f_{3}\left(s, x(s), x^{\prime}(s), D^{\beta} x(s), \int_{0}^{s} h(\xi) x(\xi) d \xi, \phi(x(s))\right) d s \mid \\
\leq & \int_{0}^{\lambda} G(t, s) \mid f_{1}\left(s, x(s), x^{\prime}(s), D^{\beta} x(s), \int_{0}^{s} h(\xi) x(\xi) d \xi, \phi(x(s))\right) \\
& -f_{1}(s, 0,0,0,0,0) \mid d s \\
& +\int_{0}^{\lambda} G(t, s)\left|f_{1}(s, 0,0,0,0,0)\right| d s \\
& +\int_{\lambda}^{\mu} G(t, s) \Phi(s) H\left(x(s), x^{\prime}(s), D^{\beta} x(s), \int_{0}^{s} h(\xi) x(\xi) d \xi, \phi(x(s))\right) d s
\end{aligned}
$$




$$
\begin{aligned}
& +\int_{\mu}^{1} G(t, s) \mid f_{3}\left(s, x(s), x^{\prime}(s), D^{\beta} x(s), \int_{0}^{s} h(\xi) x(\xi) d \xi, \phi(x(s))\right) \\
& -f_{3}(s, 0,0,0,0,0) \mid d s \\
& +\int_{\mu}^{1} G(t, s)\left|f_{3}(s, 0,0,0,0,0)\right| d s \\
\leq & \int_{0}^{\lambda} G(t, s)\left(l_{1}\|x\|+l_{2}\left\|x^{\prime}\right\|+l_{3}\left\|D^{\beta} x\right\|+l_{4}\|x\| \int_{0}^{s}|h(\xi)| d \xi+l_{5} \phi(\|x\|)\right) d s \\
& +H\left(l\|x\|_{*}, \ldots, l\|x\|_{*}\right) \int_{\lambda}^{\mu} G(t, s) \Phi(s) \\
& +\int_{\mu}^{1} G(t, s)\left(l_{1}^{\prime}\|x\|+l_{2}^{\prime}\left\|x^{\prime}\right\|+l_{3}^{\prime}\left\|D^{\beta} x\right\|\right. \\
& \left.+l_{4}^{\prime}\|x\| \int_{0}^{s}|h(\xi)| d \xi+l_{5}^{\prime} \phi(\|x\|)\right) d s \\
\leq & \frac{t}{\Gamma(\alpha-1)} \int_{0}^{\lambda}(1-s)^{\alpha-2}\left(l_{1}\|x\|+l_{2}\left\|x^{\prime}\right\|+\frac{l_{3}}{\Gamma(2-\beta)}\left\|x^{\prime}\right\|\right. \\
& \left.+l_{4} m_{0}\|x\|+l_{5} \theta_{0}\|x\|+l_{5} \theta_{1}\left\|x^{\prime}\right\|\right) d s \\
& +\frac{H\left(l\|x\|_{*}, \ldots, l\|x\|_{*}\right) t}{\Gamma(\alpha-1)} \int_{\lambda}^{\mu}(1-s)^{\alpha-2} \Phi(s) \\
& +\frac{t L}{\Gamma(\alpha-1)}\|x\|_{*}+\frac{H\left(l\|x\|_{*}, \ldots, l\|x\|_{*}\right) t}{\Gamma(\alpha-1)} \int_{\mu}^{\mu}(1-s)^{\alpha-2}\left(l_{1}^{\prime}\|x\|+l_{2}^{\prime}\left\|x^{\prime}\right\|, \frac{l_{3}^{\prime}}{\Gamma(2-\beta)}\left\|x^{\prime}\right\|+l_{4}^{\prime} m_{0}\|x\|\right. \\
& \left.+l_{5}^{\prime} \theta_{0}\|x\|+l_{5}^{\prime} \theta_{1}\left\|x^{\prime}\right\|\right) d s \\
& \frac{t-2}{\Gamma} \Phi(s) d s+\frac{t L}{\Gamma(\alpha-1)}\|x\|_{*} \\
& \\
&
\end{aligned}
$$

and so $\left\|F_{x}\right\| \leq \frac{L}{\Gamma(\alpha-1)}\|x\|_{*}+\frac{H\left(l\|x\|_{*}, \ldots, l\|x\|_{*}\right)}{\Gamma(\alpha-1)} \int_{\lambda}^{\mu}(1-s)^{\alpha-2} \Phi(s) d s+\frac{L}{\Gamma(\alpha-1)}\|x\|_{*}$. By using similar calculations, we get $\left\|F_{x}^{\prime}\right\| \leq \frac{L}{\Gamma(\alpha-1)}\|x\|_{*}+\frac{H\left(l\|x\|_{*}, \ldots, l\|x\|_{*}\right)}{\Gamma(\alpha-1)} \int_{\lambda}^{\mu}(1-s)^{\alpha-2} \Phi(s) d s+\frac{L}{\Gamma(\alpha-1)}\|x\|_{*}$. This implies that

$$
\begin{aligned}
\left\|F_{x}\right\|_{*} & =\max \left\{\left\|F_{x}\right\|,\left\|F_{x}^{\prime}\right\|\right\} \\
& \leq \frac{L}{\Gamma(\alpha-1)}\|x\|_{*}+\frac{H\left(l\|x\|_{*}, \ldots, l\|x\|_{*}\right)}{\Gamma(\alpha-1)} \int_{\lambda}^{\mu}(1-s)^{\alpha-2} \Phi(s) d s+\frac{L}{\Gamma(\alpha-1)}\|x\|_{*} \\
& <\infty
\end{aligned}
$$

This proves the claim. Since $G$ and $G^{\prime}$ are continuous with respect to $t$, it is easy to check that $F_{x}\left(t_{2}\right) \rightarrow F_{x}\left(t_{1}\right)$ as $t_{2} \rightarrow t_{1}$. By using the Arzela-Ascoli theorem, we get $\overline{T(M)}$ is relatively compact and so $F: P \rightarrow P$ is completely continuous. Since $\lim _{z \rightarrow 0^{+}} \frac{H(z, z, z, z, z)}{z}=0$, one concludes that $\lim _{\|x\|_{*} \rightarrow 0^{+}} \frac{H\left(l\|x\|_{*}, \ldots, l\|x\|_{*}\right)}{l\|x\|_{*}}=0$. Let $\epsilon>0$ be given. Choose $\delta=\delta(\epsilon)>0$ such that $\|x\|_{*}<\delta$ implies $\frac{H\left(l\|x\| x\left\|_{*}, \ldots, l\right\| x \|_{*}\right)}{l\|x\|_{*}}<\epsilon$ and so $H\left(l\|x\|_{*}, \ldots, l\|x\|_{*}\right)<\epsilon l\|x\|_{*}$. Since $\frac{L\left(1-(1-\lambda)^{\alpha-1}\right)+L^{\prime}(1-\mu)^{\alpha-1}}{\Gamma(\alpha)}<1$, there exists $\epsilon_{0}>0$ such that

$$
\frac{L\left(1-(1-\lambda)^{\alpha-1}\right)+L^{\prime}(1-\mu)^{\alpha-1}}{\Gamma(\alpha)}+\frac{\epsilon_{0} l\|\Phi\|^{*}}{\Gamma(\alpha-1)}<1,
$$


where $\|\Phi\|^{*}=\int_{\lambda}^{\mu}(1-s)^{\alpha-2} \Phi(s) d s$. Let $\delta_{0}=\delta\left(\epsilon_{0}\right)$. Define $\Omega_{1}=\left\{x \in X\right.$ s.t. $\left.\|x\|_{*}<\delta\right\}$. Then

$$
\begin{aligned}
\left|F_{x}(t)\right| \leq & \int_{0}^{\lambda} G(t, s)\left|f_{1}\left(s, x(s), x^{\prime}(s), D^{\beta} x(s), \int_{0}^{s} h(\xi) x(\xi) d \xi, \phi(x(s))\right)\right| d s \\
& +\int_{\lambda}^{\mu} G(t, s)\left|f_{2}\left(s, x(s), x^{\prime}(s), D^{\beta} x(s), \int_{0}^{s} h(\xi) x(\xi) d \xi, \phi(x(s))\right)\right| d s \\
& +\int_{\mu}^{1} G(t, s)\left|f_{3}\left(s, x(s), x^{\prime}(s), D^{\beta} x(s), \int_{0}^{s} h(\xi) x(\xi) d \xi, \phi(x(s))\right)\right| d s \\
\leq & \int_{0}^{\lambda} G(t, s)\left(l_{1}\|x\|+l_{2}\left\|x^{\prime}\right\|+\frac{l_{3}}{\Gamma(2-\beta)}\left\|x^{\prime}\right\|+l_{4} m_{0}\|x\|\right. \\
& \left.+l_{5} \theta_{0}\|x\|+l_{5} \theta_{1}\left\|x^{\prime}\right\|\right) d s \\
& +\int_{\lambda}^{\mu} G(t, s) \Phi(s) H\left(x(s), x^{\prime}(s), D^{\beta} x(s), \int_{0}^{s} h(\xi) x(\xi) d \xi, \phi(x(s))\right) d s \\
& +\int_{\mu}^{1} G(t, s)\left(l_{1}^{\prime}\|x\|+l_{2}^{\prime}\left\|x^{\prime}\right\|+\frac{l_{3}^{\prime}}{\Gamma(2-\beta)}\left\|x^{\prime}\right\|+l_{4}^{\prime} m_{0}\|x\|\right. \\
& \left.+l_{5}^{\prime} \theta_{0}\|x\|+l_{5}^{\prime} \theta_{1}\left\|x^{\prime}\right\|\right) d s \\
\leq & \frac{t L}{\Gamma(\alpha-1)}\|x\|_{*} \int_{0}^{\lambda}(1-s)^{\alpha-2} d s \\
& +\frac{t H\left(l\|x\|_{*}, l\|x\|_{*}, l\|x\|_{*}, l\|x\|_{*}, l\|x\|_{*}\right)}{\Gamma(\alpha-1)} \int_{\lambda}^{\mu}(1-s)^{\alpha-2} \Phi(s) d s \\
& +\int_{\mu}^{1} \frac{t L^{\prime}}{\Gamma(\alpha-1)}\|x\|_{*} \int_{0}^{\lambda}(1-s)^{\alpha-2} d s
\end{aligned}
$$

for all $x \in \Omega_{1}$ and $t \in[0,1]$. Hence,

$$
\left\|F_{x}\right\| \leq\left[\frac{L\left(1-(1-\lambda)^{\alpha-1}\right)+L^{\prime}(1-\mu)^{\alpha-1}}{\Gamma(\alpha)}+\frac{\epsilon_{0} l\|\Phi\|^{*}}{\Gamma(\alpha-1)}\right]\|x\|_{*} \leq\|x\|_{*} .
$$

Similarly, we get $\left\|F_{x}^{\prime}\right\| \leq\|x\|_{*}$ and so $\left\|F_{x}\right\|_{*} \leq\|x\|_{*}$. Since $\lim _{\max }\left\|x_{i}\right\| \rightarrow \infty \frac{f_{2}\left(t, x_{1} x_{2}, \ldots, x_{5}\right)}{\max \left\|x_{i}\right\|}=q(t)$, there exists $R=R(\epsilon)>0$ such that $\max \left\|x_{i}\right\|>R(\epsilon)$ implies that $\frac{f_{2}\left(t, x_{1} x_{2}, \ldots, x_{5}\right)}{\max \left\|x_{i}\right\|}>q(t)-\epsilon$ and so $f_{2}\left(t, x_{1} x_{2}, \ldots, x_{5}\right)>\left(\max \left\|x_{i}\right\|\right)(q(t)-\epsilon)$. Recall that

$$
\frac{\alpha-2}{\Gamma(\alpha)} \int_{\lambda}^{\mu}(\mu-s)^{\alpha-1} d s-\frac{\epsilon_{1}(\alpha-2)(\mu-\lambda)^{\alpha}}{\Gamma(\alpha+1)}>1 .
$$

Choose $R_{1}=R\left(\epsilon_{1}\right)>0$. Put $\Omega_{2}=\left\{x \in X:\|x\|_{*}<R_{1}\right\}$. Then

$$
\begin{aligned}
\left\|F_{x}\right\| & =\sup _{t \in[0,1]}\left|F_{x}(t)\right| \\
& \geq\left|F_{x}(\mu)\right| \\
& \geq \int_{\lambda}^{\mu} G(t, s) f_{2}\left(s, x(s), x^{\prime}(s), D^{\beta} x(s), \int_{0}^{s} h(\xi) x(\xi) d \xi, \phi(x(s))\right) d s \\
& \geq \int_{\lambda}^{\mu} \frac{(\mu-s)^{\alpha-1}(\alpha-2)}{\Gamma(\alpha)}\left(q(s)-\epsilon_{1}\right) \max \left\{\|x\|,\left\|x^{\prime}\right\|, \ldots, \| \phi(x(s) \|\} d s\right.
\end{aligned}
$$




$$
\begin{aligned}
& \geq\|x\|_{*} \int_{\lambda}^{\mu} \frac{(\mu-s)^{\alpha-1}(\alpha-2)}{\Gamma(\alpha)}\left(q(s)-\epsilon_{1}\right) d s \\
& =\|x\|_{*}\left[\int_{\lambda}^{\mu} \frac{(\mu-s)^{\alpha-1}(\alpha-2)}{\Gamma(\alpha)} q(s) d s-\epsilon_{1} \int_{\lambda}^{\mu} \frac{(\mu-s)^{\alpha-1}(\alpha-2)}{\Gamma(\alpha)} d s\right] \\
& =\|x\|_{*}\left[\frac{\alpha-2}{\Gamma(\alpha)} \int_{\lambda}^{\mu}(\mu-s)^{\alpha-1} q(s) d s-\frac{\epsilon_{1}(\alpha-2)(\mu-\lambda)^{\alpha}}{\Gamma(\alpha+1)}\right]>\|x\|_{*}
\end{aligned}
$$

for all $x \in P \cap \partial \Omega_{2}$. Hence, $\left\|F_{x}\right\|_{*} \geq\|x\|_{*}$ on $P \cap \partial \Omega_{2}$. Now by using Lemma 2.4, $F: X \rightarrow X$ has a fixed point on $P \cap\left(\Omega_{2} \backslash \Omega_{1}\right)$ which is a solution for the problem (1).

Example 3.1 Define the map $d$ on $[0.1,0.9]$ by $d(t)=\frac{1}{c(t)}$ whenever $t \in[0.1,0.9] \cap \mathbb{Q}$ where $c(t)=0$ on $[0.1,0.9] \cap \mathbb{Q}$ and $d(t)=10$ whenever $t \in[0.1,0.9] \cap \mathbb{Q}^{c}$. Now, consider the point-wise defined fractional integro-differential equation $D^{\frac{7}{2}} x(t)+f\left(t, x(t), x^{\prime}(t), D^{\frac{1}{2}} x(t)\right.$, $\left.\int_{0}^{t} x(s) d s, D^{\frac{1}{3}} x(t)\right)=0$, where

$$
f\left(t, x_{1}, x_{2}, x_{3}, x_{4}, x_{5}\right)= \begin{cases}t \sum_{i=1}^{5} x_{i} & 0 \leq t<0.1 \\ d(t) H\left(x_{1}, x_{2}, x_{3}, x_{4}, x_{5}\right) & 0.1 \leq t \leq 0.9 \\ (1-t) \sum_{i=1}^{5} x_{i} & 0.9<t \leq 1\end{cases}
$$

and $H\left(x_{1}, x_{2}, x_{3}, x_{4}, x_{5}\right)=\sum_{i=1}^{5} \frac{\left\|x_{i}\right\|^{2}}{1+\left\|x_{i}\right\|}$. Put $f_{1}\left(t, x_{1}, x_{2}, x_{3}, x_{4}, x_{5}\right)=t \sum_{i=1}^{5} x_{i}$,

$$
f_{2}\left(t, x_{1}, x_{2}, x_{3}, x_{4}, x_{5}\right)=d(t) H\left(x_{1}, x_{2}, x_{3}, x_{4}, x_{5}\right),
$$

and $f_{3}\left(t, x_{1}, x_{2}, x_{3}, x_{4}, x_{5}\right)=(1-t) \sum_{i=1}^{5} x_{i}$. Note that

$$
\begin{aligned}
& f_{1}(t, 0,0,0,0,0)=f_{3}(t, 0,0,0,0,0)=0 \\
& f_{1}\left(t, x_{1}, x_{2}, x_{3}, x_{4}, x_{5}\right)-f_{1}\left(t, y_{1}, y_{2}, y_{3}, y_{4}, y_{5}\right) \leq t \sum_{i=1}^{5}\left\|x_{i}-y_{i}\right\| \leq 0.1 \sum_{i=1}^{5}\left\|x_{i}-y_{i}\right\| \\
& \left|f_{2}\left(t, x_{1}, x_{2}, x_{3}, x_{4}, x_{5}\right)-f_{2}\left(t, y_{1}, y_{2}, y_{3}, y_{4}, y_{5}\right)\right| \\
& \quad=d(t) \sum_{i=1}^{5}\left|\frac{\left\|x_{i}\right\|^{2}}{1+\left\|x_{i}\right\|}-\frac{\left\|y_{i}\right\|^{2}}{1+\left\|y_{i}\right\|}\right| \\
& \quad=d(t) \sum_{i=1}^{5}\left|\frac{\left\|x_{i}\right\|^{2}-\left\|x_{i}\right\|^{2}\left\|y_{i}\right\|-\left\|y_{i}\right\|^{2}-\left\|y_{i}\right\|^{2}\left\|x_{i}\right\|}{\left(1+\left\|x_{i}\right\|\right)\left(1+\left\|y_{i}\right\|\right)}\right| \\
& \quad=d(t) \sum_{i=1}^{5}\left|\frac{\left\|x_{i}\right\|^{2}-\left\|y_{i}\right\|^{2}+\left\|x_{i}\right\|\left(\left\|x_{i}\right\|\left\|y_{i}\right\|-\left\|y_{i}\right\|^{2}\right)}{\left(1+\left\|x_{i}\right\|\right)\left(1+\left\|y_{i}\right\|\right)}\right| \\
& \quad=d(t) \sum_{i=1}^{5}\left|\frac{\left(\left\|x_{i}\right\|-\left\|y_{i}\right\|\right)\left(\left\|x_{i}\right\|+\left\|y_{i}\right\|\right)+\left\|x_{i}\right\|\left(\left\|x_{i}\right\|-\left\|y_{i}\right\|\right)\left\|y_{i}\right\|}{\left(1+\left\|x_{i}\right\|\right)\left(1+\left\|y_{i}\right\|\right)}\right| \\
& \quad=d(t) \sum_{i=1}^{5}\left|\frac{\left(\left\|x_{i}\right\|-\left\|y_{i}\right\|\right)\left(\left\|x_{i}\right\|+\left\|y_{i}\right\|+\left\|x_{i}\right\|\left\|y_{i}\right\|\right)}{\left\|x_{i}\right\|+\left\|y_{i}\right\|+\left\|x_{i}\right\|\left\|y_{i}\right\|+1}\right| \\
& \quad \leq d(t) \sum_{i=1}^{5}\left|\frac{\left(\left\|x_{i}\right\|-\left\|y_{i}\right\|\right)\left(\left\|x_{i}\right\|+\left\|y_{i}\right\|+\left\|x_{i}\right\|\left\|y_{i}\right\|\right)}{\left\|x_{i}\right\|+\left\|y_{i}\right\|+\left\|x_{i}\right\|\left\|y_{i}\right\|}\right|
\end{aligned}
$$




$$
\begin{aligned}
& =d(t) \sum_{i=1}^{5}\left|\left\|x_{i}\right\|-\left\|y_{i}\right\|\right| \leq d(t) \sum_{i=1}^{5}\left\|x_{i}-y_{i}\right\| \\
& :=d(t) \sum_{i=1}^{5} \Lambda_{i}\left(x_{1}-y_{1}, \ldots, x_{5}-y_{5}\right)
\end{aligned}
$$

and $f_{3}\left(t, x_{1}, x_{2}, x_{3}, x_{4}, x_{5}\right)-f_{3}\left(t, y_{1}, y_{2}, y_{3}, y_{4}, y_{5}\right) \leq 0.1 \sum_{i=1}^{5}\left\|x_{i}-y_{i}\right\|$, where $\Lambda_{i}\left(x_{1}, \ldots, x_{5}\right)=$ $\left\|x_{i}\right\|$ for $i=1, \ldots, 5$. Note that

$$
\begin{aligned}
L & =\left(l_{1}+l_{2}+\frac{l_{3}}{\Gamma(2-\beta)}+m_{0} l_{4}+\theta_{0} l_{5}+\theta_{1} l_{5}\right) \\
& =\left(0.1+0.1+\frac{0.1}{\Gamma\left(2-\frac{1}{2}\right)}+0.1+\frac{0.1}{\Gamma\left(2-\frac{1}{3}\right)}<0.4,\right. \\
L^{\prime} & =\left(l_{1}^{\prime}+l_{2}^{\prime}+\frac{l_{3}^{\prime}}{\Gamma(2-\beta)}+m_{0} l_{4}^{\prime}+\theta_{0} l_{5}^{\prime}+\theta_{1} l_{5}^{\prime}\right) \\
& =\left(0.1+0.1+\frac{0.1}{\Gamma\left(2-\frac{1}{2}\right)}+0.1+\frac{0.1}{\Gamma\left(2-\frac{1}{3}\right)}<0.4,\right.
\end{aligned}
$$

and $\lim _{z \rightarrow 0^{+}} \frac{\Lambda_{i}(z, z, z, z, z)}{z}=1:=q_{i}$ for $i=1, \ldots, 5$. Then we have

$$
\frac{L\left(1-(1-\lambda)^{\alpha-1}\right)}{\Gamma(\alpha)}+\frac{L^{\prime}}{\Gamma(\alpha)}(1-\mu)^{\alpha-1}<\frac{0.4\left(1-(1-0.1)^{\frac{5}{2}}\right)}{\Gamma\left(\frac{7}{2}\right)}+\frac{0.4(1-0.9)^{\frac{5}{2}}}{\Gamma\left(\frac{7}{2}\right)}<1
$$

and for almost all $t \in[0,1]$

$$
\begin{aligned}
q(t) & :=\lim _{\max \left\|x_{i}\right\| \rightarrow \infty} \frac{f_{2}\left(t, x_{1} x_{2}, \ldots, x_{5}\right)}{\max \left\|x_{i}\right\|}=d(t) \lim _{\max \left\|x_{i}\right\| \rightarrow \infty} \frac{\sum_{i=1}^{5} \frac{\left\|x_{i}\right\|^{2}}{1+\left\|x_{i}\right\|}}{\max \left\|x_{i}\right\|} \\
& \geq d(t) \lim _{\left\|x_{r}\right\| \rightarrow \infty} \frac{\left\|x_{i}\right\|^{2}}{\left\|x_{r}\right\|\left(1+\left\|x_{r}\right\|\right)}=d(t) \lim _{\left\|x_{r}\right\| \rightarrow \infty} \frac{\left\|x_{r}\right\|}{1+\left\|x_{r}\right\|}=d(t),
\end{aligned}
$$

where $\left\|x_{r}\right\|=\max _{1 \leq i \leq 5}\left\|x_{i}\right\|$. Thus, we obtain

$$
\frac{\alpha-2}{\Gamma(\alpha)} \int_{\lambda}^{\mu}(\mu-s)^{\alpha-2} q(s) d s \geq \frac{\frac{3}{2}}{\Gamma\left(\frac{7}{2}\right)} \int_{0.1}^{0.9} 10(0.9-s)^{\frac{3}{2}} d s>1 .
$$

Now, by using Theorem 3.2, the problem has a solution.

Theorem 3.3 Let $\left[\lambda, \mu, f=\left(f_{1}, f_{2}, f_{3}\right)\right]$ with $f_{1}(s, 0,0,0,0,0)=f_{3}(t, 0,0,0,0,0)=0$ for all $s \in[0, \lambda]$ and $t \in[\mu, 1]$. Assume that there exist nonnegative functions $a \in L^{1}[0, \lambda], c \in$ $L^{1}[\mu, 1]$ and $b_{1}, \ldots, b_{5}:[\lambda, \mu] \rightarrow \mathbb{R}$ with $\hat{b}_{i}:=(1-t)^{\alpha-2} b_{i}(t) \in L^{1}[\lambda, \mu](i=1, \ldots, 5)$ such that $\left|f_{1}\left(t, x_{1}, \ldots, x_{5}\right)-f_{1}\left(t, y_{1}, \ldots, y_{5}\right)\right| \leq a(t) \sum_{i=1}^{5}\left\|x_{i}-y_{i}\right\|$,

$$
\left|f_{2}\left(t, x_{1}, \ldots, x_{5}\right)-f_{2}\left(t, y_{1}, \ldots, y_{5}\right)\right| \leq \sum_{i=1}^{5} b_{i}(t)\left\|x_{i}-y_{i}\right\|
$$

and $\left|f_{3}\left(t, x_{1}, \ldots, x_{5}\right)-f_{3}\left(t, y_{1}, \ldots, y_{5}\right)\right| \leq c(t) \sum_{i=1}^{5}\left\|x_{i}-y_{i}\right\|$ for all $x_{1}, \ldots, x_{5}, y_{1}, \ldots, y_{5} \in X$ and almost all $t \in[0,1]$. Suppose that there exist a natural number $n_{0}$ and nonnegative functions $\phi_{1}, \ldots, \phi_{n_{0}}$ with $\hat{\phi}_{i}:=(1-t)^{\alpha-2} \phi_{i}(t) \in L^{1}[\lambda, \mu]$ and nonnegative and nondecreasing 
with respect to all components maps $\Lambda_{1}, \ldots, \Lambda_{n_{0}}: X^{5} \rightarrow[0, \infty)$ with $\lim _{z \rightarrow 0^{+}} \frac{\Lambda_{i}(z, z, z, z, z)}{z}=0$ such that $\left|f_{2}\left(t, x_{1}, \ldots, x_{5}\right)\right| \leq \sum_{i=1}^{n_{0}} \phi_{i} \Lambda_{i}\left(x_{1}, \ldots, x_{5}\right)$ for all $\left(x_{1}, \ldots, x_{5}\right) \in X$ and almost all $t \in[\lambda, \mu]$. If $\left(2+\frac{1}{\Gamma(2-\beta)}+m_{0}+\theta_{0}+\theta_{1}\right)\left(\|a\|_{[0, \lambda]}+\sum_{i=1}^{5}\left\|\hat{b_{i}}\right\|+(1-\mu)^{\alpha-2}\|c\|_{[1, \mu]}\right)<\Gamma(\alpha-1)$, then the problem (1) has a solution.

Proof First we show that $F$ is a continuous map on $X$. Let $x_{1}, x_{2} \in X$ and $t \in[0,1]$. Then

$$
\begin{aligned}
& \left|F_{x_{1}}(t)-F_{x_{2}}(t)\right| \leq \int_{0}^{\lambda} G(t, s) \mid f_{1}\left(s, x_{1}(s), x_{1}^{\prime}(s), D^{\beta} x_{1}(s), \int_{0}^{s} h(\xi) x_{1}(\xi) d \xi, \phi\left(x_{1}(s)\right)\right) \\
& -f_{1}\left(s, x_{2}(s), x_{2}^{\prime}(s), D^{\beta} x_{2}(s), \int_{0}^{s} h(\xi) x_{2}(\xi) d \xi, \phi\left(x_{2}(s)\right)\right) \mid d s \\
& +\int_{\lambda}^{\mu} G(t, s) \mid f_{2}\left(s, x_{1}(s), x_{1}^{\prime}(s), D^{\beta} x_{1}(s), \int_{0}^{s} h(\xi) x_{1}(\xi) d \xi, \phi\left(x_{1}(s)\right)\right) d s \\
& -f_{2}\left(s, x_{2}(s), x_{2}^{\prime}(s), D^{\beta} x_{2}(s), \int_{0}^{s} h(\xi) x_{2}(\xi) d \xi, \phi\left(x_{2}(s)\right)\right) \mid d s \\
& +\int_{\mu}^{1} G(t, s) \mid f_{3}\left(s, x_{1}(s), x_{1}^{\prime}(s), D^{\beta} x_{1}(s), \int_{0}^{s} h(\xi) x_{1}(\xi) d \xi, \phi\left(x_{1}(s)\right)\right) d s \\
& -f_{3}\left(s, x_{2}(s), x_{2}^{\prime}(s), D^{\beta} x_{2}(s), \int_{0}^{s} h(\xi) x_{2}(\xi) d \xi, \phi\left(x_{2}(s)\right)\right) \mid d s \\
& \leq \int_{0}^{\lambda} \frac{t(1-s)^{\alpha-2}}{\Gamma(\alpha-1)} a(s)\left(\left|x_{1}(s)-x_{2}(s)\right|+\left|x_{1}^{\prime}(s)-x_{2}^{\prime}(s)\right|+\left|D^{\beta}\left(x_{1}-x_{2}\right)(s)\right|\right. \\
& \left.+\int_{0}^{s}\left|x_{1}(\xi)-x_{2}(\xi)\right| d \xi+\left|\phi\left(x_{1}(s)-x_{2}(s)\right)\right|\right) d s \\
& +\int_{\lambda}^{\mu} \frac{t(1-s)^{\alpha-2}}{\Gamma(\alpha-1)}\left(b_{1}(s)\left|x_{1}(s)-x_{2}(s)\right|+b_{2}(s)\left|x_{1}^{\prime}(s)-x_{2}^{\prime}(s)\right|\right. \\
& +b_{3}(s)\left|D^{\beta}\left(x_{1}-x_{2}\right)(s)\right| \\
& \left.+b_{4}(s) \int_{0}^{s}\left|x_{1}(\xi)-x_{2}(\xi)\right| d \xi+b_{5}(s)\left|\phi\left(x_{1}(s)-x_{2}(s)\right)\right|\right) d s \\
& +\int_{\mu}^{1} \frac{t(1-s)^{\alpha-2}}{\Gamma(\alpha-1)} c(s)\left(\left|x_{1}(s)-x_{2}(s)\right|\right. \\
& +\left|x_{1}^{\prime}(s)-x_{2}^{\prime}(s)\right|+\left|D^{\beta}\left(x_{1}-x_{2}\right)(s)\right| \\
& \left.+\int_{0}^{s}\left|x_{1}(\xi)-x_{2}(\xi)\right| d \xi+\left|\phi\left(x_{1}(s)-x_{2}(s)\right)\right|\right) d s \\
& \leq \frac{t}{\Gamma(\alpha-1)} \int_{0}^{\lambda}(1-s)^{\alpha-2} a(s)\left(\left\|x_{1}-x_{2}\right\|+\left\|x_{1}^{\prime}-x_{2}^{\prime}\right\|+\frac{\left\|x_{1}^{\prime}-x_{2}^{\prime}\right\|}{\Gamma(2-\beta)}\right. \\
& \left.+m_{0}\left\|x_{1}-x_{2}\right\|+\theta_{0}\left\|x_{1}-x_{2}\right\|+\theta_{1}\left\|x_{1}^{\prime}-x_{2}^{\prime}\right\|\right) d s \\
& +\frac{t}{\Gamma(\alpha-1)} \int_{\lambda}^{\mu}(1-s)^{\alpha-2}\left(b_{1}(s)\left\|x_{1}-x_{2}\right\|+b_{2}(s)\left\|x_{1}^{\prime}-x_{2}^{\prime}\right\|\right. \\
& +b_{3}(s) \frac{\left\|x_{1}^{\prime}-x_{2}^{\prime}\right\|}{\Gamma(2-\beta)}+b_{4}(s) m_{0}\left\|x_{1}-x_{2}\right\| \\
& \left.+b_{5}(s)\left(\theta_{0}\left\|x_{1}-x_{2}\right\|+\theta_{1}\left\|x_{1}^{\prime}-x_{2}^{\prime}\right\|\right)\right) d s
\end{aligned}
$$




$$
\begin{aligned}
& +\frac{t}{\Gamma(\alpha-1)} \int_{\mu}^{1}(1-s)^{\alpha-2} c(s)\left(\left\|x_{1}-x_{2}\right\|+\left\|x_{1}^{\prime}-x_{2}^{\prime}\right\|+\frac{\left\|x_{1}^{\prime}-x_{2}^{\prime}\right\|}{\Gamma(2-\beta)}\right. \\
& \left.+m_{0}\left\|x_{1}-x_{2}\right\|+\theta_{0}\left\|x_{1}-x_{2}\right\|+\theta_{1}\left\|x_{1}^{\prime}-x_{2}^{\prime}\right\|\right) d s \\
& \leq \frac{t\left(2+\frac{1}{\Gamma(2-\beta)}+m_{0}+\theta_{0}+\theta_{1}\right)}{\Gamma(\alpha-1)}\left\|x_{1}-x_{2}\right\|_{*} \int_{0}^{\lambda}(1-s)^{\alpha-2} a(s) d s \\
& \quad+\frac{t\left(2+\frac{1}{\Gamma(2-\beta)}+m_{0}+\theta_{0}+\theta_{1}\right)}{\Gamma(\alpha-1)}\left\|x_{1}-x_{2}\right\|_{*} \sum_{i=1}^{5} \int_{\lambda}^{\mu}(1-s)^{\alpha-2} b_{i}(s) d s \\
& \quad+\frac{t\left(2+\frac{1}{\Gamma(2-\beta)}+m_{0}+\theta_{0}+\theta_{1}\right)}{\Gamma(\alpha-1)}\left\|x_{1}-x_{2}\right\|_{*} \int_{\mu}^{1}(1-s)^{\alpha-2} c(s) d s \\
& \leq \frac{t\left(2+\frac{1}{\Gamma(2-\beta)}+m_{0}+\theta_{0}+\theta_{1}\right)}{\Gamma(\alpha-1)}\left\|x_{1}-x_{2}\right\|_{*}\left[\int_{0}^{\lambda} a(s) d s\right. \\
& \left.\quad+\sum_{i=1}^{5} \int_{\lambda}^{\mu}(1-s)^{\alpha-2} b_{i}(s) d s+\int_{\mu}^{1} c(s) d s\right]
\end{aligned}
$$

and so

$$
\begin{aligned}
\left\|F_{x_{1}}-F_{x_{2}}\right\| \leq & \frac{\left(2+\frac{1}{\Gamma(2-\beta)}+m_{0}+\theta_{0}+\theta_{1}\right)}{\Gamma(\alpha-1)}\left[\|a\|_{[0, \lambda]}\right. \\
& \left.+\sum_{i=1}^{5}\left\|\hat{b}_{i}\right\|_{[\lambda, \mu]}+\|c\|_{[\mu, 1]}\right]\left\|x_{1}-x_{2}\right\|_{*} .
\end{aligned}
$$

By using similar calculations, we get

$$
\begin{aligned}
\mid F_{x_{1}}^{\prime}( & t)-F_{x_{2}}^{\prime}(t) \mid \\
\leq & \int_{0}^{\lambda} \frac{\partial G}{\partial t}(t, s) \mid f_{1}\left(s, x_{1}(s), x_{1}^{\prime}(s), D^{\beta} x_{1}(s), \int_{0}^{s} h(\xi) x_{1}(\xi) d \xi, \phi\left(x_{1}(s)\right)\right) \\
& -f_{1}\left(s, x_{2}(s), x_{2}^{\prime}(s), D^{\beta} x_{2}(s), \int_{0}^{s} h(\xi) x_{2}(\xi) d \xi, \phi\left(x_{2}(s)\right)\right) \mid d s \\
& +\int_{\lambda}^{\mu} \frac{\partial G}{\partial t}(t, s) \mid f_{2}\left(s, x_{1}(s), x_{1}^{\prime}(s), D^{\beta} x_{1}(s), \int_{0}^{s} h(\xi) x_{1}(\xi) d \xi, \phi\left(x_{1}(s)\right)\right) d s \\
& -f_{2}\left(s, x_{2}(s), x_{2}^{\prime}(s), D^{\beta} x_{2}(s), \int_{0}^{s} h(\xi) x_{2}(\xi) d \xi, \phi\left(x_{2}(s)\right)\right) \mid d s \\
& +\int_{\mu}^{1} \frac{\partial G}{\partial t}(t, s) \mid f_{3}\left(s, x_{1}(s), x_{1}^{\prime}(s), D^{\beta} x_{1}(s), \int_{0}^{s} h(\xi) x_{1}(\xi) d \xi, \phi\left(x_{1}(s)\right)\right) d s \\
& -f_{3}\left(s, x_{2}(s), x_{2}^{\prime}(s), D^{\beta} x_{2}(s), \int_{0}^{s} h(\xi) x_{2}(\xi) d \xi, \phi\left(x_{2}(s)\right)\right) \mid d s \\
\leq & \int_{0}^{\lambda} \frac{t(1-s)^{\alpha-2}}{\Gamma(\alpha-1)} a(s)\left(\left|x_{1}(s)-x_{2}(s)\right|+\left|x_{1}^{\prime}(s)-x_{2}^{\prime}(s)\right|+\left|D^{\beta}\left(x_{1}-x_{2}\right)(s)\right|\right. \\
& \left.+\int_{0}^{s}\left|x_{1}(\xi)-x_{2}(\xi)\right| d \xi+\left|\phi\left(x_{1}(s)-x_{2}(s)\right)\right|\right) d s
\end{aligned}
$$




$$
\begin{aligned}
& +\int_{\lambda}^{\mu} \frac{t(1-s)^{\alpha-2}}{\Gamma(\alpha-1)}\left(b_{1}(s)\left|x_{1}(s)-x_{2}(s)\right|+b_{2}(s)\left|x_{1}^{\prime}(s)-x_{2}^{\prime}(s)\right|\right. \\
& +b_{3}(s)\left|D^{\beta}\left(x_{1}-x_{2}\right)(s)\right| \\
& \left.+b_{4}(s) \int_{0}^{s}\left|x_{1}(\xi)-x_{2}(\xi)\right| d \xi+b_{5}(s)\left|\phi\left(x_{1}(s)-x_{2}(s)\right)\right|\right) d s \\
& +\int_{\mu}^{1} \frac{t(1-s)^{\alpha-2}}{\Gamma(\alpha-1)} c(s)\left(\left|x_{1}(s)-x_{2}(s)\right|+\left|x_{1}^{\prime}(s)-x_{2}^{\prime}(s)\right|+\left|D^{\beta}\left(x_{1}-x_{2}\right)(s)\right|\right. \\
& \left.+\int_{0}^{s}\left|x_{1}(\xi)-x_{2}(\xi)\right| d \xi+\left|\phi\left(x_{1}(s)-x_{2}(s)\right)\right|\right) d s \\
& \leq \frac{t\left(2+\frac{1}{\Gamma(2-\beta)}+m_{0}+\theta_{0}+\theta_{1}\right)}{\Gamma(\alpha-1)}\left\|x_{1}-x_{2}\right\|_{*} \int_{0}^{\lambda}(1-s)^{\alpha-2} a(s) d s \\
& +\frac{t\left(2+\frac{1}{\Gamma(2-\beta)}+m_{0}+\theta_{0}+\theta_{1}\right)}{\Gamma(\alpha-1)}\left\|x_{1}-x_{2}\right\|_{*} \sum_{i=1}^{5} \int_{\lambda}^{\mu}(1-s)^{\alpha-2} b_{i}(s) d s \\
& +\frac{t\left(2+\frac{1}{\Gamma(2-\beta)}+m_{0}+\theta_{0}+\theta_{1}\right)}{\Gamma(\alpha-1)}\left\|x_{1}-x_{2}\right\|_{*} \int_{\mu}^{1}(1-s)^{\alpha-2} c(s) d s \\
& \leq \frac{t\left(2+\frac{1}{\Gamma(2-\beta)}+m_{0}+\theta_{0}+\theta_{1}\right)}{\Gamma(\alpha-1)}\left\|x_{1}-x_{2}\right\|_{*}\left[\int_{0}^{\lambda} a(s) d s\right. \\
& \left.+\sum_{i=1}^{5} \int_{\lambda}^{\mu}(1-s)^{\alpha-2} b_{i}(s) d s+\int_{\mu}^{1} c(s) d s\right]
\end{aligned}
$$

and so

$$
\begin{aligned}
\left\|F_{x_{1}}^{\prime}-F_{x_{2}}^{\prime}\right\| \leq & \frac{\left(2+\frac{1}{\Gamma(2-\beta)}+m_{0}+\theta_{0}+\theta_{1}\right)}{\Gamma(\alpha-1)} \\
& \times\left[\|a\|_{[0, \lambda]}+\sum_{i=1}^{5}\left\|\hat{b}_{i}\right\|_{[\lambda, \mu]}+\|c\|_{[\mu, 1]}\right]\left\|x_{1}-x_{2}\right\|_{*} .
\end{aligned}
$$

This implies that

$$
\begin{aligned}
\left\|F_{x_{1}}-F_{x_{2}}\right\|_{*} \leq & \frac{\left(2+\frac{1}{\Gamma(2-\beta)}+m_{0}+\theta_{0}+\theta_{1}\right)}{\Gamma(\alpha-1)} \\
& \times\left[\|a\|_{[0, \lambda]}+\sum_{i=1}^{5}\left\|\hat{b}_{i}\right\|_{[\lambda, \mu]}+\|c\|_{[\mu, 1]}\right]\left\|x_{1}-x_{2}\right\|_{*}
\end{aligned}
$$

and so $F_{x_{1}} \rightarrow F_{x_{2}}$ in $X$ as $x_{2} \rightarrow x_{1}$. Thus, $F$ is continuous on $X$. We have $\lim _{z \rightarrow 0^{+}} \frac{\Lambda_{i}(z, z, z, z, z)}{z}=$ $0, \lim _{z \rightarrow 0^{+}} \frac{\Lambda_{i}(l z, l, l, l z, l, l, l z)}{z}=0$, where $l=\max \left\{1, \frac{1}{\Gamma(2-\beta)}, m_{0}, \theta_{0}+\theta_{1}\right\}$. Let $\epsilon>0$ be given. Choose $\delta_{i}:=\delta_{i}(\epsilon)>0$ such that $0<z \leq \delta_{i}$ implies that $\lim _{z \rightarrow 0^{+}} \frac{\Lambda_{i}(l z, l z, l z, l z, l z)}{z}<\epsilon$ for $1 \leq i \leq n_{0}$. Hence, $\Lambda_{i}(l z, l z, l z, l z, l z)<\epsilon z$ for $0<z \leq \delta_{i}$ and so $\Lambda_{i}(l z, l z, l z, l z, l z)<\epsilon z$ for all $1 \leq i \leq n_{0}$ and $z \in(0 . \delta]$, where $\delta:=\delta(\epsilon)=\min _{1 \leq i \leq n_{0}}\left\{\delta_{i}\right\}$. Since

$$
\left(2+\frac{1}{\Gamma(2-\beta)}+m_{0}+\theta_{0}+\theta_{1}\right)\left(\|a\|_{[0, \lambda]}+(1-\mu)^{\alpha-2}\|c\|_{[1, \mu]}\right)<\Gamma(\alpha-1)
$$


there exists $\epsilon_{0}>0$ such that

$$
\left(2+\frac{1}{\Gamma(2-\beta)}+m_{0}+\theta_{0}+\theta_{1}\right)\left(\|a\|_{[0, \lambda]}+(1-\mu)^{\alpha-2}\|c\|_{[1, \mu]}\right)+\epsilon_{0} \sum_{i=1}^{n_{0}}\left\|\hat{\phi}_{i}\right\|_{[\lambda, \mu]}<\Gamma(\alpha-1) .
$$

Let $r=\delta\left(\epsilon_{0}\right)$. Then $\Lambda_{i}(l z, l z, l z, l z, l z)<\epsilon_{0} z$ for all $1 \leq i \leq n_{0}$ and for $z \in(0, r]$. Put $C=$ $\left\{x \in X:\|x\|_{*}<r\right\}$. Define the map $\alpha: X^{2} \rightarrow[0, \infty)$ by $\alpha(x, y)=1$ whenever $x, y \in C$ and $\alpha(x, y)=0$ otherwise. We show that $F$ is $\alpha$-admissible. Let $x, y \in X$ be such that $\alpha(x, y) \geq 1$. Then $x, y \in C,\|x\|_{*}<r$ and $\|y\|_{*}<r$. Let $t \in[0,1]$. Then we have

$$
\begin{aligned}
\left|F_{x}(t)\right| \leq & \int_{0}^{\lambda} G(t, s)\left|f_{1}\left(s, x(s), x^{\prime}(s), D^{\beta} x(s), \int_{0}^{s} h(\xi) x(\xi) d \xi, \phi(x(s))\right)\right| d s \\
& +\int_{\lambda}^{\mu} G(t, s)\left|f_{2}\left(s, x(s), x^{\prime}(s), D^{\beta} x(s), \int_{0}^{s} h(\xi) x(\xi) d \xi, \phi(x(s))\right)\right| d s \\
& +\int_{\mu}^{1} G(t, s)\left|f_{3}\left(s, x(s), x^{\prime}(s), D^{\beta} x(s), \int_{0}^{s} h(\xi) x(\xi) d \xi, \phi(x(s))\right)\right| d s \\
\leq & \frac{t}{\Gamma(\alpha-1)} \int_{0}^{\lambda}(1-s)^{\alpha-2} \mid f_{1}\left(s, x(s), x^{\prime}(s), D^{\beta} x(s), \int_{0}^{s} h(\xi) x(\xi) d \xi, \phi(x(s))\right) \\
& -f_{1}(s, 0,0,0,0,0)\left|d s+\frac{t}{\Gamma(\alpha-1)} \int_{0}^{\lambda}(1-s)^{\alpha-2}\right| f_{1}(s, 0,0,0,0,0) \mid d s \\
& +\frac{t}{\Gamma(\alpha-1)} \int_{\lambda}^{\mu}(1-s)^{\alpha-2} \sum_{i=1}^{n_{0}} \phi_{i}(s) \Lambda_{i}\left(x(s), x^{\prime}(s), D^{\beta} x(s),\right. \\
& \left.\int_{0}^{s} h(\xi) x(\xi) d \xi, \phi(x(s))\right) d s \\
& +\frac{t}{\Gamma(\alpha-1)} \int_{\mu}^{1}(1-s)^{\alpha-2} \mid f_{3}\left(s, x(s), x^{\prime}(s), D^{\beta} x(s), \int_{0}^{s} h(\xi) x(\xi) d \xi, \phi(x(s))\right) \\
& \left.+m_{0}\|x\|+\theta_{0}\|x\|+\theta_{1}\|x\|\right) d s \\
& -f_{3}(s, 0,0,0,0,0) \mid d s \\
& +\frac{t}{\Gamma(\alpha-1)} \int_{\lambda}^{\mu}(1-s)^{\alpha-2} \sum_{i=1}^{n_{0}} \phi_{i}(s) \Lambda_{i}\left(\|x\|+\left\|x^{\prime}\right\|+\frac{\left\|x^{\prime}\right\|}{\Gamma(2-\beta)}\right. \\
& +\frac{t}{\Gamma(\alpha-1)} \int_{0}^{\lambda}(1-s)^{\alpha-2}\left|f_{3}(s, 0,0,0,0,0)\right| d s \\
& \frac{t}{\Gamma(\alpha-1)} \int_{0}^{\lambda}(1-s)^{\alpha-2} a(s)\left(\|x\|+\left\|x^{\prime}\right\|+\frac{\left\|x^{\prime}\right\|}{\Gamma(2-\beta)}\right. \\
& \left.+m_{0}\|x\|+\theta_{0}\|x\|+\theta_{1}\|x\|\right) d s \\
& +\theta_{1}(1-s)^{\alpha-2} c(s)\left(\|x\|+\left\|x^{\prime}\right\|+\frac{\left\|x^{\prime}\right\|}{\Gamma(2-\beta)}\right. \\
& \left.+\theta_{1} \| x\right) d s \\
& \\
&
\end{aligned}
$$




$$
\begin{aligned}
\leq & \frac{t}{\Gamma(\alpha-1)}\left(\left[2+\frac{1}{\Gamma(2-\beta)}+m_{0}+\theta_{0}+\theta_{1}\right]\|x\|_{*} \int_{0}^{\lambda} \sup (1-s)^{\alpha-2} a(s) d s\right. \\
& +\sum_{i=1}^{n_{0}} \int_{\lambda}^{\mu}(1-s)^{\alpha-2} \phi_{i}(s) \Lambda_{i}\left(l\|x\|_{*}, l\|x\|_{*}, l\|x\|_{*}, l\|x\|_{*}, l\|x\|_{*}\right) d s \\
& \left.+\left[2+\frac{1}{\Gamma(2-\beta)}+m_{0}+\theta_{0}+\theta_{1}\right]\|x\|_{*} \int_{\mu}^{1} \sup (1-s)^{\alpha-2} c(s) d s\right) \\
\leq & \frac{1}{\Gamma(\alpha-1)}\left(\left[2+\frac{1}{\Gamma(2-\beta)}+m_{0}+\theta_{0}+\theta_{1}\right]\left(\|a\|_{[0, \lambda]}+(1-\mu)^{\alpha-2}\|c\|_{[\mu, 1]}\right) r\right. \\
& \left.+\sum_{i=1}^{n_{0}} \Lambda_{i}(l r, l r, l r, l r, l r) \int_{\lambda}^{\mu} \hat{\phi}_{i}(s) d s\right) \\
= & \frac{1}{\Gamma(\alpha-1)}\left(\left[2+\frac{1}{\Gamma(2-\beta)}+m_{0}+\theta_{0}+\theta_{1}\right]\left(\|a\|_{[0, \lambda]}+(1-\mu)^{\alpha-2}\|c\|_{[\mu, 1]}\right) r\right. \\
& \left.+\epsilon_{0} \sum_{i=1}^{n_{0}}\left\|\hat{\phi}_{i}\right\|\right) r \\
& \left.+\sum_{i=1}^{n_{0}}\left\|\hat{\phi}_{i}\right\| \Lambda_{i}(l r, l r, l r, l r, l r)\right) \\
\leq & \frac{1}{\Gamma(\alpha-1)}\left(\left[2+\frac{1}{\Gamma(2-\beta)}+m_{0}+\theta_{0}+\theta_{1}\right]\left(\|a\|_{[0, \lambda]}+(1-\mu)^{\alpha-2}\|c\|_{[\mu, 1]}\right)\right. \\
& \frac{1}{\Gamma(\alpha-1)} \Gamma(\alpha-1) r=r,
\end{aligned}
$$

and so $\left\|F_{x}\right\|<r$. Similarly one can prove that $\left\|F_{x}^{\prime}\right\|<r$ and so $\left\|F_{x}\right\|_{*}=\max \left\{\left\|F_{x}\right\|,\left\|F_{x}^{\prime}\right\|\right\}<r$. Hence, $F_{x} \in C$ and by same reason $F_{y} \in C$. This implies that $\alpha\left(F_{x}, F_{y}\right) \geq 1$ and so $F$ is $\alpha$ admissible. Also, $\alpha\left(x_{0}, F_{x_{0}}\right) \geq 1$ for all $x_{0} \in C$ (note that $C$ is nonempty). Let $x, y \in X$ and $t \in[0,1]$. Then we have

$$
\begin{aligned}
\left|F_{x}(t)-F_{y}(t)\right| \leq & \int_{0}^{\lambda} G(t, s) \mid f_{1}\left(s, x(s), x^{\prime}(s), D^{\beta} x(s), \int_{0}^{s} h(\xi) x(\xi) d \xi, \phi(x(s))\right) \\
& -f_{1}\left(s, y(s), y^{\prime}(s), D^{\beta} y(s), \int_{0}^{s} h(\xi) y(\xi) d \xi, \phi(y(s))\right) \mid d s \\
& +\int_{\lambda}^{\mu} G(t, s) \mid f_{2}\left(s, x(s), x^{\prime}(s), D^{\beta} x(s), \int_{0}^{s} h(\xi) x(\xi) d \xi, \phi(x(s))\right) d s \\
& -f_{2}\left(s, y(s), y^{\prime}(s), D^{\beta} y(s), \int_{0}^{s} h(\xi) y(\xi) d \xi, \phi(y(s))\right) \mid d s \\
& +\int_{\mu}^{1} G(t, s) \mid f_{3}\left(s, x(s), x^{\prime}(s), D^{\beta} x(s), \int_{0}^{s} h(\xi) x(\xi) d \xi, \phi(x(s))\right) d s \\
& \left.-f_{3}(s, y), y^{\prime}(s), D^{\beta} y(s), \int_{0}^{s} h(\xi) y(\xi) d \xi, \phi(y(s))\right) \mid d s \\
\leq & \frac{t}{\Gamma(\alpha-1)} \int_{0}^{\lambda}(1-s)^{\alpha-2} a(s)\left(\|x-y\|+\left\|x^{\prime}-y^{\prime}\right\|+\left\|D^{\beta}(x-y)\right\|\right.
\end{aligned}
$$




$$
\begin{aligned}
& \left.+\int_{0}^{s}|h(\xi)|\|x-y\| d \xi+\phi(\|x-y\|)\right) d s \\
& +\frac{t}{\Gamma(\alpha-1)} \int_{\lambda}^{\mu}(1-s)^{\alpha-2}\left(b_{1}(s)\|x-y\|+b_{2}(s)\left\|x^{\prime}-y^{\prime}\right\|\right. \\
& \left.+b_{3}(s)\left(D^{\beta}\|x-y\|\right)+b_{4}(s) \int_{0}^{s}|h(\xi)|\|x-y\| d \xi+b_{5}(s) \phi(\|x-y\|)\right) d s \\
& +\frac{t}{\Gamma(\alpha-1)} \int_{\mu}^{1}(1-s)^{\alpha-2} c(s)\left(\|x-y\|+\left\|x^{\prime}-y^{\prime}\right\|+\left\|D^{\beta}(x-y)\right\|\right. \\
& \left.+\int_{0}^{s}|h(\xi)|\|x-y\| d \xi+\phi(\|x-y\|)\right) d s \\
& \leq \frac{t}{\Gamma(\alpha-1)} \int_{0}^{\lambda}(1-s)^{\alpha-2} a(s)\left(\|x-y\|+\left\|x^{\prime}-y^{\prime}\right\|+\frac{\left\|x^{\prime}-y^{\prime}\right\|}{\Gamma(2-\beta)}\right. \\
& \left.+m_{0}\|x-y\|+\theta_{0}\|x-y\|+\theta_{1}\left\|x^{\prime}-y^{\prime}\right\|\right) d s \\
& +\frac{t}{\Gamma(\alpha-1)} \int_{\lambda}^{\mu}(1-s)^{\alpha-2}\left(b_{1}(s)\|x-y\|+b_{2}(s)\left\|x^{\prime}-y^{\prime}\right\|\right. \\
& +b_{3}(s) \frac{\left\|x^{\prime}-y^{\prime}\right\|}{\Gamma(2-\beta)}+b_{4}(s) m_{0}\|x-y\| \\
& \left.+b_{5}(s)\left(\theta_{0}\|x-y\|+\theta_{1}\left\|x^{\prime}-y^{\prime}\right\|\right)\right) d s \\
& +\frac{t}{\Gamma(\alpha-1)} \int_{\mu}^{1}(1-s)^{\alpha-2} c(s)\left(\|x-y\|+\left\|x^{\prime}-y^{\prime}\right\|+\frac{\left\|x^{\prime}-y^{\prime}\right\|}{\Gamma(2-\beta)}\right. \\
& \left.+m_{0}\|x-y\|+\theta_{0}\|x-y\|+\theta_{1}\left\|x^{\prime}-y^{\prime}\right\|\right) d s \\
& \leq \frac{t\left(2+\frac{1}{\Gamma(2-\beta)}+m_{0}+\theta_{0}+\theta_{1}\right)}{\Gamma(\alpha-1)}\|x-y\|_{*}\left[\int_{0}^{\lambda} \sup (1-s)^{\alpha-2} a(s) d s\right. \\
& \left.+\sum_{i=1}^{5} \int_{\lambda}^{\mu}(1-s)^{\alpha-2} b_{i}(s) d s+\int_{\mu}^{1} \sup (1-s)^{\alpha-2} c(s) d s\right] \\
& \leq \frac{1}{\Gamma(\alpha-1)}\left(2+\frac{1}{\Gamma(2-\beta)}+m_{0}+\theta_{0}+\theta_{1}\right) \\
& \times\left(\|a\|_{[0, \lambda]}+\sum_{i=1}^{5}\left\|\hat{b}_{i}\right\|+(1-\mu)^{\alpha-2}\|c\|_{[1, \mu]}\right)\|x-y\|_{*} \\
& :=\psi\left(\|x-y\|_{*}\right) \text {. }
\end{aligned}
$$

Similarly, one can show that $\left\|F_{x}^{\prime}-F_{y}^{\prime}\right\| \leq \psi\left(\|x-y\|_{*}\right)$ and so $\alpha(x, y)\left\|F_{x}-F_{y}\right\|_{*} \leq \psi(d(x, y))$ for all $x, y \in X$. We have

$$
\frac{1}{\Gamma(\alpha-1)}\left(2+\frac{1}{\Gamma(2-\beta)}+m_{0}+\theta_{0}+\theta_{1}\right)\left(\|a\|_{[0, \lambda]}+\sum_{i=1}^{5}\left\|\hat{b}_{i}\right\|+(1-\mu)^{\alpha-2}\|c\|_{[1, \mu]}\right)<1
$$

$\psi \in \Psi$. By using Lemma 2.2, $F$ has a fixed point which is a solution for the problem (1). 
Example 3.2 Consider the problem $D^{\frac{9}{2}} x(t)+f\left(t, x(t), x^{\prime}(t), D^{\frac{1}{2}} x(t), \int_{0}^{t} x(\xi) d \xi, I^{\frac{1}{3}} x(t)\right)=0$, where

$$
f\left(t, x_{1}, \ldots, x_{5}\right)= \begin{cases}f_{1}\left(t, x_{1}, \ldots, x_{5}\right):=\sin t\left(\sum_{i=1}^{5}\left\|x_{i}\right\|\right) & t \in[0,0.2), \\ f_{2}\left(t, x_{1}, \ldots, x_{5}\right):=\frac{0.2}{p(t)} \sum_{i=1}^{5} \frac{\left\|x_{i}\right\|^{2}}{1+\left\|x_{i}\right\|} & t \in[0.2,0.7], \\ f_{3}\left(t, x_{1}, \ldots, x_{5}\right):=t\left(\sum_{i=1}^{5}\left\|x_{i}\right\|\right) & t \in[0.7,1],\end{cases}
$$

and $p(t)=0$ whenever $t \in[0.2,0.07] \cap \mathcal{Q}$ and $p(t)=\sqrt{t}$ whenever $t \in[0.2,0.07] \cap \mathcal{Q}^{c}$. Put $a(t)=\sin t, b_{1}(t)=\cdots=b_{5}(t)=\frac{1}{p(t)}$ and $c(t)=t$ for all $t$. Note that

$$
\begin{aligned}
& \left|f_{1}\left(t, x_{1}, \ldots, x_{5}\right)-f_{1}\left(t, y_{1}, \ldots, y_{5}\right)\right|=\sin t\left|\sum_{i=1}^{5}\left\|x_{i}\right\|-\left\|y_{i}\right\|\right| \leq \sin t \sum_{i=1}^{5}\left\|x_{i}-y_{i}\right\|, \\
& \left|f_{2}\left(t, x_{1}, \ldots, x_{5}\right)-f_{2}\left(t, y_{1}, \ldots, y_{5}\right)\right| \\
& =\frac{0.2}{p(t)}\left|\sum_{i=1}^{5} \frac{\left\|x_{i}\right\|^{2}}{1+\left\|x_{i}\right\|}-\frac{\left\|y_{i}\right\|^{2}}{1+\left\|y_{i}\right\|}\right| \\
& =\frac{0.2}{p(t)}\left|\sum_{i=1}^{5} \frac{\left\|x_{i}\right\|^{2}+\left\|x_{i}\right\|^{2}\left\|y_{i}\right\|-\left\|x_{i}\right\|\left\|y_{i}\right\|^{2}-\left\|y_{i}\right\|^{2}}{\left(1+\left\|x_{i}\right\|\right)\left(1+\left\|y_{i}\right\|\right)}\right| \\
& =\frac{0.2}{p(t)}\left|\sum_{i=1}^{5} \frac{\left(\left\|x_{i}\right\|+\left\|y_{i}\right\|\right)\left(\left\|x_{i}\right\|-\left\|y_{i}\right\|\right)+\left\|x_{i}\right\|\left(\left\|x_{i}\right\|-\left\|y_{i}\right\|\right)\left\|y_{i}\right\|}{\left(1+\left\|x_{i}\right\|\right)\left(1+\left\|y_{i}\right\|\right)}\right| \\
& =\frac{0.2}{p(t)}\left|\sum_{i=1}^{5} \frac{\left(\left\|x_{i}\right\|-\left\|y_{i}\right\|\right)\left(\left\|x_{i}\right\|+\left\|y_{i}\right\|+\left\|x_{i}\right\|\left\|y_{i}\right\|\right)}{\left(1+\left\|x_{i}\right\|+\left\|y_{i}\right\|+\left\|x_{i}\right\|\left\|y_{i}\right\|\right.}\right| \\
& \quad \leq \frac{0.2}{p(t)}\left|\sum_{i=1}^{5}\left\|x_{i}\right\|-\left\|y_{i}\right\|\right| \leq \frac{0.2}{p(t)} \sum_{i=1}^{5}\left\|x_{i}-y_{i}\right\| .
\end{aligned}
$$

Define $\Lambda_{i}\left(x_{1}, \ldots, x_{5}\right)=\frac{\left\|x_{i}\right\|^{2}}{1+\left\|x_{i}\right\|}$ for $i=1, \ldots, 5$. Then $\lim _{z \rightarrow 0^{+}} \frac{\Lambda_{i}(z, z, z, z, z)}{z}=0$ for all $i$. Put $b_{i}(t)=$ $\phi_{i}(t)=\frac{0.2}{p(t)}$ for all $i, n_{0}=5$ and $\beta=\frac{1}{2}$. Since $\left|\int_{0}^{t} x(\xi) d \xi\right| \leq t\|x\| \leq\|x\|$, put $m_{0}=1$. Since $\left|I^{\frac{1}{3}} x(t)\right|=\left|\frac{1}{\Gamma\left(\frac{1}{3}\right)} \int_{0}^{t}(t-s)^{\frac{1}{3}-1} x(s) d s\right| \leq \frac{1}{\Gamma\left(\frac{1}{3}\right)} \int_{0}^{t}\left|(t-s)^{\frac{1}{3}-1} x(s)\right| d s \leq \frac{\|x\|}{\Gamma\left(\frac{1}{3}\right)} \int_{0}^{t} \frac{d s}{(t-s)^{\frac{2}{3}}} \leq \frac{\|x\|}{\Gamma\left(\frac{1}{2}\right)}$, we put $\theta_{0}=\frac{1}{\Gamma\left(\frac{1}{3}\right)}$ and $\theta_{1}=0$. Note that $\|a\|_{[0, \lambda]}=\int_{0}^{0.2} \sin t d t \leq 0.02,\left\|\hat{b}_{i}\right\|_{[\lambda, \mu]}=\int_{0.2}^{0.7} \frac{0.2}{\sqrt{t}} d t \leq 0.08$, $\|c\|_{[\mu, 1]}=\int_{0.7}^{1} t d t=0.045$ and

$$
\begin{aligned}
(2 & \left.+\frac{1}{\Gamma(2-\beta)}+m_{0}+\theta_{0}+\theta_{0}+\theta_{1}\right)\left(\|a\|_{[0, \lambda]}+\sum_{i=1}^{5}\left\|\hat{b}_{i}\right\|+(1-\mu)^{\alpha-2}\|c\|_{[\mu, 1]}\right) \\
& \leq\left(2+\frac{1}{\Gamma\left(\frac{3}{2}\right)}+1+\frac{1}{\Gamma\left(\frac{1}{2}\right)}\right)\left(0.02+\sum_{i=1}^{5} 0.8+(1-0.7)^{\frac{7}{2}} 0.045\right) \\
& \leq\left(3+\frac{2}{\sqrt{\pi}}+\frac{1}{\sqrt{\pi}}\right)(0.421)<\Gamma\left(\frac{7}{2}\right)=\Gamma(\alpha-1) .
\end{aligned}
$$

Now by using Theorem 3.3, the problem has a solution. 


\section{Conclusions}

Most natural phenomena include crisis and it is important we could model this type phenomena. Researchers are going to use fractional integro-differential equations for modeling of crisis phenomena. In this work, we investigate the existence of solutions for a three steps crisis integro-differential equation by considering this assumption that the second step is a point-wise defined singular fractional differential equation, while the first and third parts have natural treatments.

\section{Competing interests}

The authors declare that they have no competing interests.

\section{Authors' contributions}

The main idea of this paper was proposed by the third author. All authors read and approved the final manuscript.

\section{Author details}

${ }^{1}$ Department of Mathematics, Cankaya University, Ankara, Turkey. ${ }^{2}$ Institute of Space Sciences, Bucharest, Romania. ${ }^{3}$ Department of Mathematics, Azarbaijan Shahid Madani University, Tabriz, Iran. ${ }^{4}$ Department of Medical Research, China Medical University Hospital, China Medical University, Taichung, Taiwan. ${ }^{5}$ Department of Mathematics, Islamic Azad University, Mehran Branch, Mehran, Iran.

\section{Publisher's Note}

Springer Nature remains neutral with regard to jurisdictional claims in published maps and institutional affiliations.

Received: 3 January 2018 Accepted: 1 April 2018 Published online: 16 April 2018

\section{References}

1. Berezowski, M.: Crisis phenomenon in a chemical reactor with recycle. Chem. Eng. Sci. 101, 451-453 (2013)

2. Cheraghlou, A.M.: The aftermath of financial crises: a look on human and social wellbeing. World Dev. 87, 88-106 (2016)

3. Ivanov, I., Kabaivanov, S., Bogdanova, B.: Stock market recovery from the 2008 financial crisis: the differences across Europe. Res. Int. Bus. Finance 37, 360-374 (2016)

4. Naseradinmousavi, P., Nataraj, C.: Transient chaos and crisis phenomena in butterfly valves driven by solenoid actuators. Commun. Nonlinear Sci. Numer. Simul. 17, 4336-4345 (2012)

5. Novelli, E.M., Gladwin, M.T.: Crises in sickle cell disease. Chest 149, 1082-1093 (2016)

6. Surtaev, A., Pavlenko, A.: Observation of boiling heat transfer and crisis phenomena in falling water film at transient heating. Int. J. Heat Mass Transf. 74, 342-352 (2014)

7. Surtaev, A.S., Pavlenko, A.N., Kuznetsov, D.V., Kalita, V.I., Komlev, D.I., Ivannikov, A.Y., Radyuk, A.A.: Heat transfer and crisis phenomena at pool boiling of liquid nitrogen on the surfaces with capillary-porous coatings. Int. J. Heat Mass Transf. 108, 146-155 (2017)

8. Zhao, L., Li, W., Cai, X.: Structure and dynamics of stock market in times of crisis. Phys. Lett. A 380, 654-666 (2016)

9. Alfaro, M., Coville, J.: Propagation phenomena in monostable integro-differential equations: acceleration or not? J. Differ. Equ. 263(9), 5727-5758 (2017)

10. Calleja, R.C., Humphries, A.R., Krauskopf, B.: Resonance phenomena in a scalar delay differential equation with two state-dependent delays. SIAM J. Appl. Dyn. Syst. 16(3), 1474-1513 (2017)

11. Chian, A.C.L., Rempel, E.L., Macau, E.E., Rosa, R.R., Christiansen, F.: High-dimensional interior crisis in the Kuramoto-Sivashinsky equation. Phys. Rev. E 65(3), 035203 (2002)

12. Franaszek, M., Nabaglo, A.: General case of crisis-induced intermittency in the Duffing equation. Phys. Lett. A 178(1-2), 85-91 (1993)

13. Gsponer, A., Hurni, J.P.: Lanczos's equation as a way out of the spin $3 / 2$ crisis? Higher spins, QCD and beyond. Hadron. J. 26(3-4), 327-350 (2003)

14. Almeida, R., Bastos, B.R.O., Monteiro, M.T.T.: Modeling some real phenomena by fractional differential equations. Math Methods Appl. Sci. 39(16), 4846-4855 (2016)

15. Agarwal, R.P., O'Regan, D., Stanek, S.: Positive solutions for Dirichlet problems of singular nonlinear fractional differential equations. J. Math. Anal. Appl. 371, 57-68 (2010)

16. Agarwal, R.P., O'Regan, D., Stanek, S.: Positive solutions for mixed problems of singular fractional differential equations. Math. Nachr. 285(1), 27-41 (2012)

17. Bai, Z., Qui, T.: Existence of positive solution for singular fractional differential equation. Appl. Math. Comput. 215, 2761-2767 (2009)

18. Rezapour, S., Shabibi, M.: A singular fractional fractional differential equation with Riemann-Liouville integral boundary condition. J. Adv. Math. Stud. 8(1), 80-88 (2015)

19. Shabibi, M., Postolache, M., Rezapour, S., Vaezpour, S.M.: Investigation of a multi-singular pointwise defined fractional integro-differential equation. J. Math. Anal. 7(5), 61-77 (2016)

20. Stanek, S.: The existence of positive solutions of singular fractional boundary value problems. Comput. Math. Appl. 62, 1379-1388 (2011)

21. Tatar, N.: An impulsive nonlinear singular version of the Gronwall-Bihari inequality. J. Inequal. Appl. 2006, Article ID 84561 (2006) 
22. Podlubny, I.: Fractional Differential Equations. Academic Press, San Diego (1999)

23. Samet, B., Vetro, C., Vetro, P.: Fixed point theorems for $\alpha$ - $\psi$-contractive type mappings. Nonlinear Anal. 75 , 2154-2165 (2012)

24. Samko, S.G., Kilbas, A.A., Marichev, O.I.: Fractional Integral and Derivative: Theory and Applications. Gordon \& Breach New York (1993)

25. Krasnoselskii, M.A.: Positive Solutions of Operator Equations. Noordhoff, Groningen (1964)

Submit your manuscript to a SpringerOpen ${ }^{\circ}$ journal and benefit from:

- Convenient online submission

- Rigorous peer review

- Open access: articles freely available online

- High visibility within the field

Retaining the copyright to your article

Submit your next manuscript at $\boldsymbol{\triangleright}$ springeropen.com 